\title{
Poplar Short Rotation Coppice Plantations under Mediterranean Conditions: The Case of Spain
}

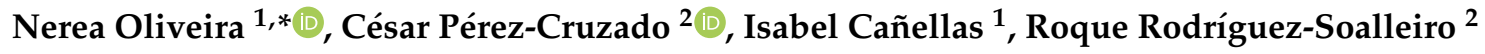 \\ and Hortensia Sixto ${ }^{1}$ \\ 1 Forest Research Centre, National Institute for Agriculture and Food Research and Technology (INIA-CIFOR), \\ Crta. de la Coruña km 7.5, E-28040 Madrid, Spain; canellas@inia.es (I.C.); sixto@inia.es (H.S.) \\ 2 Sustainable Forest Management Group, University of Santiago de Compostela, C/Benigno Ledo s/n, \\ E-27002 Lugo, Spain; cesar.cruzado@usc.es (C.P.-C.); roque.rodriguez@usc.es (R.R.-S.) \\ * Correspondence: oliveira.nerea@inia.es
}

Received: 15 November 2020; Accepted: 14 December 2020; Published: 17 December 2020

\begin{abstract}
Developing a circular bioeconomy based on the sustainable use of biological resources, such as biomass, seems to be the best way of responding to the challenges associated with global change. Among the many sources, short rotation forest crops are an essential instrument for obtaining quality biomass with a predictable periodicity and yield, according to the areas of cultivation. This review aims to provide an overview of available knowledge on short rotation coppice Populus spp. plantations under Mediterranean conditions and specifically in Spain, in order to identify not only the status, but also the future prospects, for this type of biomass production. The analysis of available information was conducted by taking into consideration the following aspects: Genetic plant material; plantation design, including densities, rotation lengths and the number of rotations, and mixtures; management activities, including irrigation, fertilization, and weed control; yield prediction; biomass characterization; and finally, an evaluation of the sustainability of the plantation and ecosystem services provided. Despite advances, there is still much to be done if these plantations are to become a commercial reality in some Mediterranean areas. To achieve this aim, different aspects need to be reconsidered, such as irrigation, bearing in mind that water restrictions represent a real threat; the specific adaptation of genetic material to these conditions, in order to obtain a greater efficiency in resource use, as well as a greater resistance to pests and diseases or tolerance to abiotic stresses such as drought and salinity; rationalizing fertilization; quantifying and valuing the ecosystem services; the advance of more reliable predictive models based on ecophysiology; the specific characterization of biomass for its final use (bioenergy/bioproducts); technological improvements in management and harvesting; and finally, improving the critical aspects detected in environmental, energy, and economic analyses to achieve profitable and sustainable plantations under Mediterranean conditions.
\end{abstract}

Keywords: biomass; Populus; SRC (Short Rotation Coppice); short rotation woody crops; sustainability; Mediterranean conditions; management; review

\section{Introduction}

The challenges associated with climate change, along with the changing paradigm for both economic development and the energy model, have been crystallized into a Green Deal for Europe [1]. In this context, the use, production, and utilization of biomass are undoubtedly some of the main issues, very much related to the need to redirect the linear economy towards a circular bioeconomy based on the use of sustainable biological resources [2]. The Innovation Strategy for Sustainable Growth: A Bioeconomy for Europe [3] and its later revision [4] establish the bioeconomy as the general framework and key factor for achieving green, sustainable growth in Europe. In harmony with the 
European strategy, while also taking into account the national possibilities, the Spanish Strategy for the Bioeconomy [5] and the current draft Law on Climate Change and Energy Transition [6] have been published.

Development in this area must take into consideration the potential of natural resources, including biomass. The options of using existing biomass in forests, agricultural residues, or the production of new biomass from crops planted specifically for this purpose are all seen as key to the development of the bioeconomy [3], not only for economic reasons, but also with environmental and social considerations in mind. In Spain, the Strategic Research and Innovation Agenda in relation to biomass has also recently been presented [7]. In this context, biomass is a highly valued resource for both bioenergy production and bioproducts, thus contributing towards addressing the abovementioned global challenges.

There are various sources of forest biomass, which can be differentiated into the following: (i) That derived from forest management activities associated with timber exploitation; (ii) residues resulting from silvicultural operations apart from timber exploitation; (iii) biomass derived from the forestry industry; and lastly, (iv) dedicated forest crops specifically designed for the production of woody biomass.

The first category includes logging residues and small diameter or crooked logs which are used for energy production (pellets, wood chips, or firewood). The first and third categories are the type of biomass mainly used in Spain today. The Spanish Renewable Energy Association (APPA) has repeatedly pointed to the underutilization of wood resources at a national level, as only $41 \%$ of the annual wood increment is currently being used, which is notably below the average of $60-70 \%$ for Europe [8].

However, if the amount of wood resources has been constantly increasing since the 1960 's, what is the point of developing specific forestry crops? Firstly, these crops are seen as being of particular importance in terms of their potential contribution to the efficient diversification of biomass sources [9-11]. Secondly, of all biomass sources, forest crops specifically designed for woody biomass production are those most readily managed in terms of both time and space, with a predictable periodicity and yield. Thirdly, biomass from forest crops can contribute to generating and stabilizing the biomass market.

The aim of this study is to assess the advances made in poplar short rotation coppice (SRC) plantations over recent years, focusing on specific advances in Mediterranean conditions under irrigation (Spain) within the global context and to identify the areas of research where progress still needs to be made. The current state-of-the-art in biomass production from poplar SRC under Mediterranean conditions is addressed, highlighting both the strengths and weaknesses.

Therefore, this paper is structured around the following: (i) A global vision of SRC plantations focusing on poplar as one of the most suitable species in Mediterranean areas; (ii) the state-of-the-art of these plantations at a global level, while focusing on the progress made in Spain; and finally, (iii) conclusions drawn under Mediterranean conditions, with a particular emphasis on the weaknesses identified and the short- and long-term lines of research needed to address them.

\section{Short Rotation Forest Crops for the Production of Biomass: The Populus Genus}

Despite the different dedicated energy crops, the current agenda [7] only considers herbaceous and woody lignocellulosic crops for more diverse uses, which also include bioproducts.

Fast growing species are used in SRC, employing intensive or semi-intensive techniques [12], with coppicing cycles of between 2 and 8 years until stool productivity declines, which normally occurs after 15 years [13,14], depending on the site quality.

Biomass from SRC may become essential as an addition to the biomass provided by forests, contributing towards meeting the demands of European industry and assuring market stability [15]. This is probably linked to the need to find spatiotemporal complementarity in biomass resources, contributing to matching the supply with the demand; this circumstance is already a commercial reality in many parts of the world [16]. The suitability of such biomass is also linked to the 
intrinsic characteristics of its production and management [17], such as the abundance of improved, highly adaptable genetic material; high rate of successful rooting; good juvenile growth; and its resprouting capacity, among others [18-20]. Other traits associated with its end use are also deemed to be important, such as a low chlorine $(\mathrm{Cl})$ and sulfur $(\mathrm{S})$ content, low ash content, and high lignin content [21-24], among others.

SRC have been found to provide ecosystem services seldom sufficiently quantified and contrasted, such as air cleaning; the control of erosion or flooding in certain areas [25-27]; mitigation of the effects of climate change through carbon fixing in foliar or root biomass fractions [14,28,29]; increases of the biodiversity in agricultural environments [30-33]; and even soil decontamination [34-36], including mining reclamation $[37,38]$. From a social perspective, woody crops contribute towards the creation of employment in rural areas, given that these crops provide an opportunity to make use of poor, marginal, or surplus agricultural land [39]. Finally, the use of biomass from SRC helps to reduce the pressure on natural forests by providing a raw material much demanded by society and therefore by industry.

Despite this, to achieve sustainability in the implementation of SRC, it is important to consider the impact of land use changes in areas where these plantations compete with agricultural crops for land, as well as aspects related to water consumption in areas with limited water resources. Therefore, it is necessary to define the limitations in order to guarantee the sustainability of these crops [40]. Many of these aspects can probably be dealt with through the use of biotechnology to achieve improvements, or by using circular economy techniques such as wastewater reuse [41-43].

In Spain, the interest in producing biomass from SRC dates back to the mid-80's [44-46], coinciding to a large extent with the crisis in the oil sector. However, it was around 2000 when initiatives to increase sources of renewable energy at a global scale, particularly in Europe, provided the impetus to explore possibilities for the production of biomass as a renewable resource. The climatic, edaphic, and demographic characteristics of some parts of the country are suitable for the cultivation of SRC, with an expected high productivity [47] exceeding that obtained in other European countries. However, because of the Mediterranean climatic conditions, such plantations are only viable with the use of irrigation. In areas with an Atlantic climate, the conditions for SRC are also suitable, with limitations such as the orographic characteristics, which may complicate the intensive silviculture applied in this type of plantation, or the limitations associated with certain species due to soil acidity.

Although many studies have focused on the selection of vegetal material best adapted to given areas of Spain, there is still a long way to go with regards to identifying the interaction between the genotype and the environment, which is a determining factor in the success or failure of SRC.

There are many woody species potentially cultivable for biomass production. In general, they are fast growing broadleaf species with a high re-sprouting capacity.

In Europe, the Salicaceae family (Populus spp. and Salix spp.) presents the greatest developments on an industrial level. Plantations based on species and hybrids of Populus are well-established in both central and southern Europe, with examples in Germany [48], the United Kingdom [13], the Czech Republic [49], Bulgaria [50], Serbia [51], Poland [52], and France [53], as well as in Mediterranean regions, mainly Italy [54] and Spain [55]. In northern Europe, where Salix spp. has been the predominant species for this purpose, poplar cultivation is beginning to attract interest, with different trials being established to evaluate its potential $[56,57]$.

To a large extent, Populus species are at the forefront of biomass production because of the highly efficient breeding programs in different countries, many of which are located in Europe [58]. These programs are favored due to the very broad genetic base with which to work in terms of traits linked to cultivation and wood properties $[59,60]$, but also due to knowledge of the genome sequence [61] and the relatively short breeding cycles. Other factors include a high capacity for vegetative reproduction and their rapid growth rate.

The importance of cultivating Salicaceae for biomass production at a global scale is reflected by the abundance of information gathered over the last decade by the International Poplar Commission 
(statutory body within the FAO), which has specifically covered the subject in one of its working groups [62]. The International Catalogue of Base Materials of Populus for obtaining forest reproductive material contains 358 entries, among which several are specifically referenced for biomass production ('Boiano-4', 'Baldo', 'Hunneghem', and 'Raspalje'). Many more are at preliminary stages for inclusion and have been put forward for this use ('AF2', 'AF8', 'A4A', 'Monviso', 'Muur', 'Orion', 'Oudenberg', 'Sirio', and 'Vesten'). Within the framework of the EU-POP project, more than 17 genotypes are currently being characterized as biomass producers in a multi-environment trial in which ten European countries are taking part, including Spain [63].

In addition to the genotypes that have been catalogued (or are in the cataloguing phase) for this purpose, there are others that have been identified for their wood production, but which also seem to possess suitable characteristics for the production of biomass. Figure 1 shows the main genotypes that are being tested or planted for commercial purposes in some European countries.

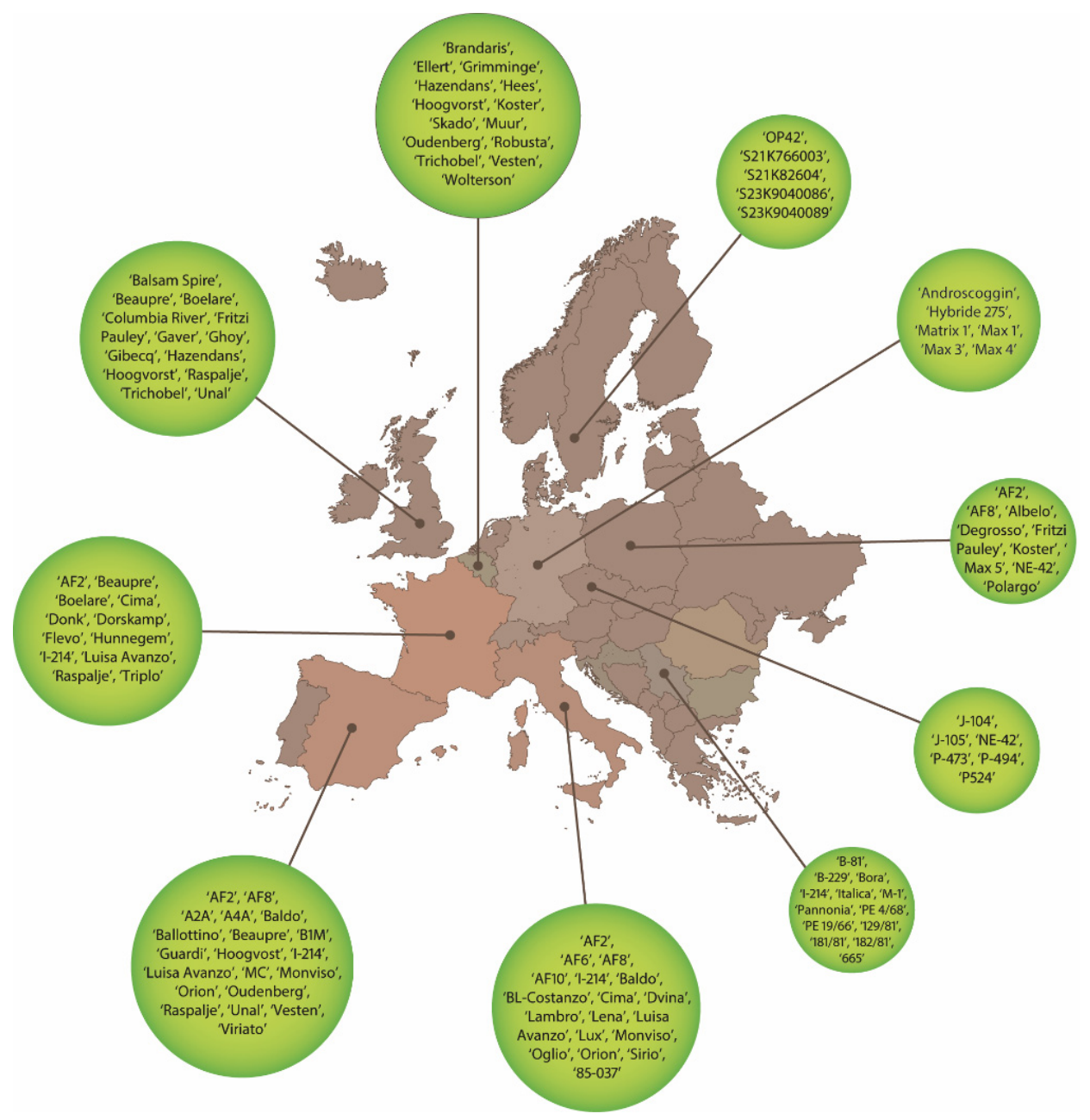

Figure 1. Some of the main genotypes planted for biomass purposes in each country are represented on a European map.

In Spain, the main species of interest at a commercial or pre-commercial level are those belonging to the genera Populus spp. and Eucalyptus spp. The cultivation of species and hybrids of Populus for industrial wood (veneer) is well-established in many areas of the country [64]. Although poplar production plantations in Spain represent less than $1 \%$ of the tree-covered forest area (approximately $100,000 \mathrm{ha}$ ), in some provinces, these plantations account for more than $50 \%$ of the harvested wood, which is around $40 \%$ of the economic value of roundwood cuttings. In the province of Castilla $y$ Leon, for example, poplar is the forest species with the highest economic value $[65,66]$. However, 
regarding poplar in SRC, there is only a token presence in Spain, with it occupying around 50 ha at its peak. The potential land at a national level corresponds to irrigated agricultural marginal land for food production with the edaphoclimatic requirements for the species [47]. Currently, the irrigated agricultural land in Spain is 3.8 Mha [67].

The genotypes for planting should be included in the European Catalogue of Base Material for Populus reproductive material. There is also a Spanish National Catalogue of Base Material for forest reproductive material of the Populus genus in qualified and controlled categories comprising 24 commercial quality genotypes (Table 1). Some of this material, which is well-adapted to the specific Spanish Mediterranean conditions, could also be of interest for biomass production. Most of the genotypes already tested or currently being tested are listed in Figure 1, and include materials from both the European and Spanish lists, as well as some that have not yet been catalogued. The potential of Populus is explained to a large degree by the availability of material adapted to existing conditions, along with appropriate knowledge relating to the management of the species in many parts of the country $[68,69]$.

Table 1. Genotypes included in the National Catalogue of Base Materials for the production of forest reproductive materials related to the genus Populus L.

\begin{tabular}{|c|c|c|c|}
\hline Parentage & & Section & Genotypes \\
\hline P. $\times$ canadensis Mönch & $\mathrm{D} \times \mathrm{N}$ & Aigeiros & $\begin{array}{l}\text { '2000 Verde', 'Agathe F' a, } \\
\text { 'E-298' a ,'Branagesi', 'B-1M', } \\
\text { 'Canadá Blanco', ,'Dorskamp', } \\
\text { 'Flevo', 'Guardi', 'I-214' b', } \\
\text { 'Campeador' b ,'I-454/40', } \\
\text { 'Luisa Avanzo', 'MC', 'Triplo' }\end{array}$ \\
\hline $\begin{array}{l}\text { P. deltoides W. Bartram } \\
\text { ex Marshall }\end{array}$ & $\mathrm{D}$ & Aigeiros & 'Lux', 'Viriato' \\
\hline P. $\times$ generosa Henry & $\mathrm{T} \times \mathrm{D}$ & Tacamahaca $\times$ Aigeiros & $\begin{array}{c}\text { 'Beaupre', 'Boelare', 'Raspalje', } \\
\text { 'Unal', 'USA 49-177' }\end{array}$ \\
\hline $\begin{array}{l}\text { P. } \times \text { generosa Henry } \\
\quad \times \text { P. alba } \mathrm{L} .\end{array}$ & $(\mathrm{T} \times \mathrm{D}) \times \mathrm{A}$ & $\begin{array}{c}\text { (Tacamahaca } \times \text { Aigeiros }) \\
\times \text { Populus }\end{array}$ & 'I-114/69' \\
\hline P. nigra L. & $\mathrm{N}$ & Aigeiros & $\begin{array}{l}\text { 'Tr 56/75', 'Bordils', } \\
\text { 'Lombardo leones' }\end{array}$ \\
\hline
\end{tabular}

$\mathrm{D}$ is P. deltoides; N is P. nigra; $\mathrm{T}$ is P. trichocarpa; A is P. alba; ' 'Agathe $\mathrm{F}^{\prime}=$ ' $\mathrm{E}-298^{\prime}$; and ${ }^{\mathrm{b}}$ ' $\mathrm{I}-214^{\prime}$ = 'Campeador'. Order of 24/06/1992, Order APA/544/2003 of 06/032003, Resolution of 07/07/2006 of the Dirección General de Agricultura, and Resolution of 07/11/2011 of the Dirección General de Recursos Agrícolas y Ganaderos.

In relation to Eucalyptus, the cultivation of some species and hybrids for the production of biomass has been significant in certain areas of the country, reaching production values of $14.6 \mathrm{Mg} \mathrm{ha}^{-1}$ year $^{-1}$ in the case of Eucalyptus globulus Labill. and $21.5 \mathrm{Mg} \mathrm{ha}^{-1}$ year ${ }^{-1}$ for Eucalyptus nitens (Deane and Maiden) Maiden [70] in Atlantic areas without irrigation, whereas the development of similar yields in southern Spain would need irrigation.

Some genotypes of Eucalyptus exhibit the additional advantage of presenting an acceptable degree of tolerance to drought conditions [71,72], although the high levels of production are associated with scenarios where droughts do not occur or where irrigation is used. One of the main differences with respect to poplar is that Eucalyptus is an evergreen species, so harvested trees include twigs and even leaves, yielding biomass with a high ash content.

Furthermore, trials with other potentially usable species have taken place in recent years, leading to differing results. In this regard, at a Mediterranean scale, there have been experimental trials with Ulmus pumila L. [73], Robinia pseudoacacia L. [74-77], hybrids of Salix spp. [78-81], Platanus $\times$ hispanica Mill. ex Münchh [77], and different cultivars of Paulownia spp. [82,83], with the latter displaying severe adaptation problems to the climatic conditions of the Mediterranean area (early frosts and flooding) [84]. Although some of these may be of interest in terms of adding diversity to the area of 
crop development, perhaps the main limitation of most of these species stems from the fact that there is a lack of improved genetic material, which is necessary for allowing their use in different environments.

\section{Lines of Progress: State of the Art}

Sustainable improvement in biomass production from poplar SRC crops requires advances to be made in different aspects, which have been summarized in Figure 2. An overall vision of the current situation of each of these aspects is provided in this section.

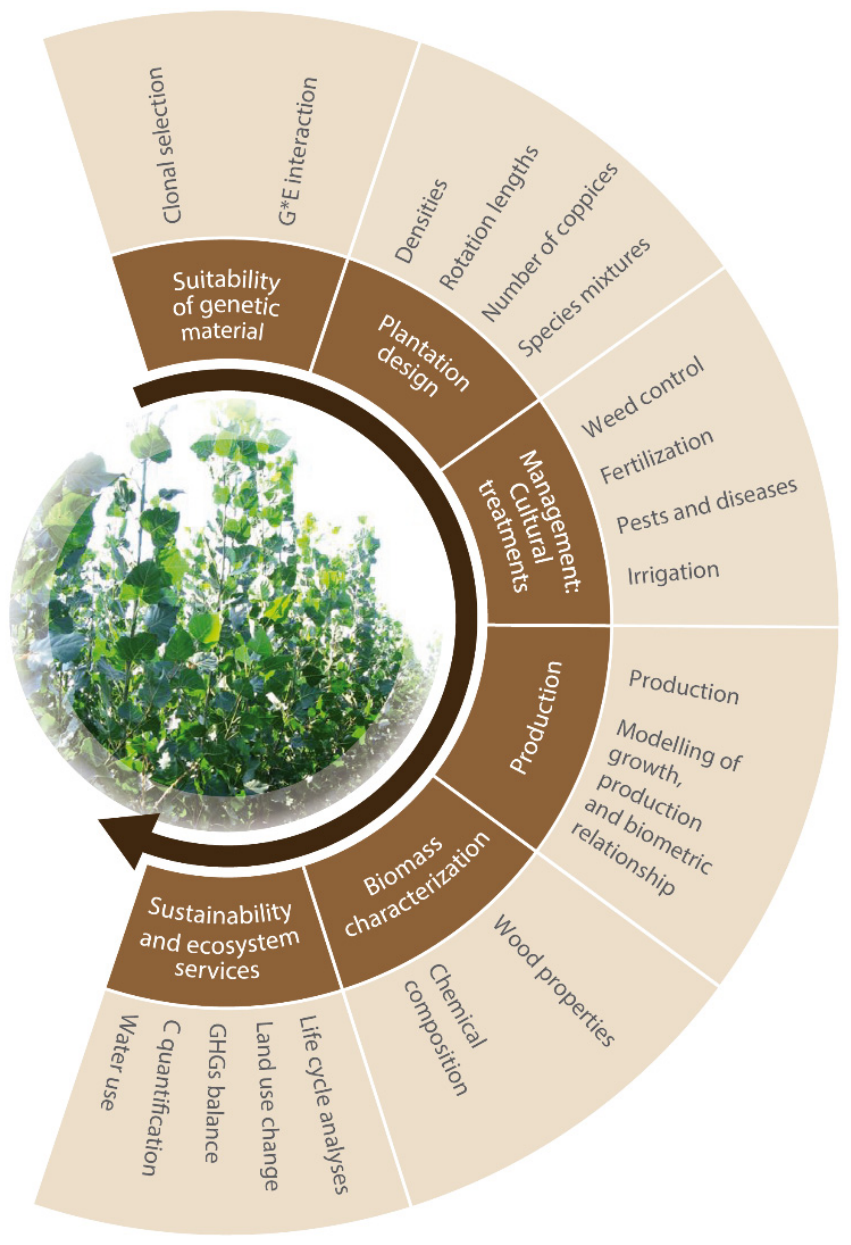

Figure 2. Lines of action for improving short rotation coppice-managed woody crops.

Literature searches for the combination of the terms Populus or poplar, and short rotation or energy crop, were conducted using Web of Science (WOS, Core collection of Web of Science), which is one of the main journal databases. There were no restrictions in terms of the year of publication or language. Figure 3 shows the number of documents per country (including only the top 30 countries) found in WOS, totaling 2185 documents. From these searches, documents from Spain were filtered and it was found that some of them were not properly classified in the database. Out of the 112 documents relating to Spain (ranked 10th) found in the WOS, only 83 were included.

In this review, we took into account not only the documents found in this database, but also other scientific publications belonging to Journal Citations Report (JCR) indexed journals, as well as numerous pieces of available gray literature. All this information was identified by tracing back papers cited in the references of the identified studies and reviewing publications by scientists who have worked or are currently working on poplar short rotation in Spain. In any case, given the abundance of existing information and the difficulties associated with the use of diverse terminology, there may be certain literature that we are not aware of, and therefore has not been considered in this review. 


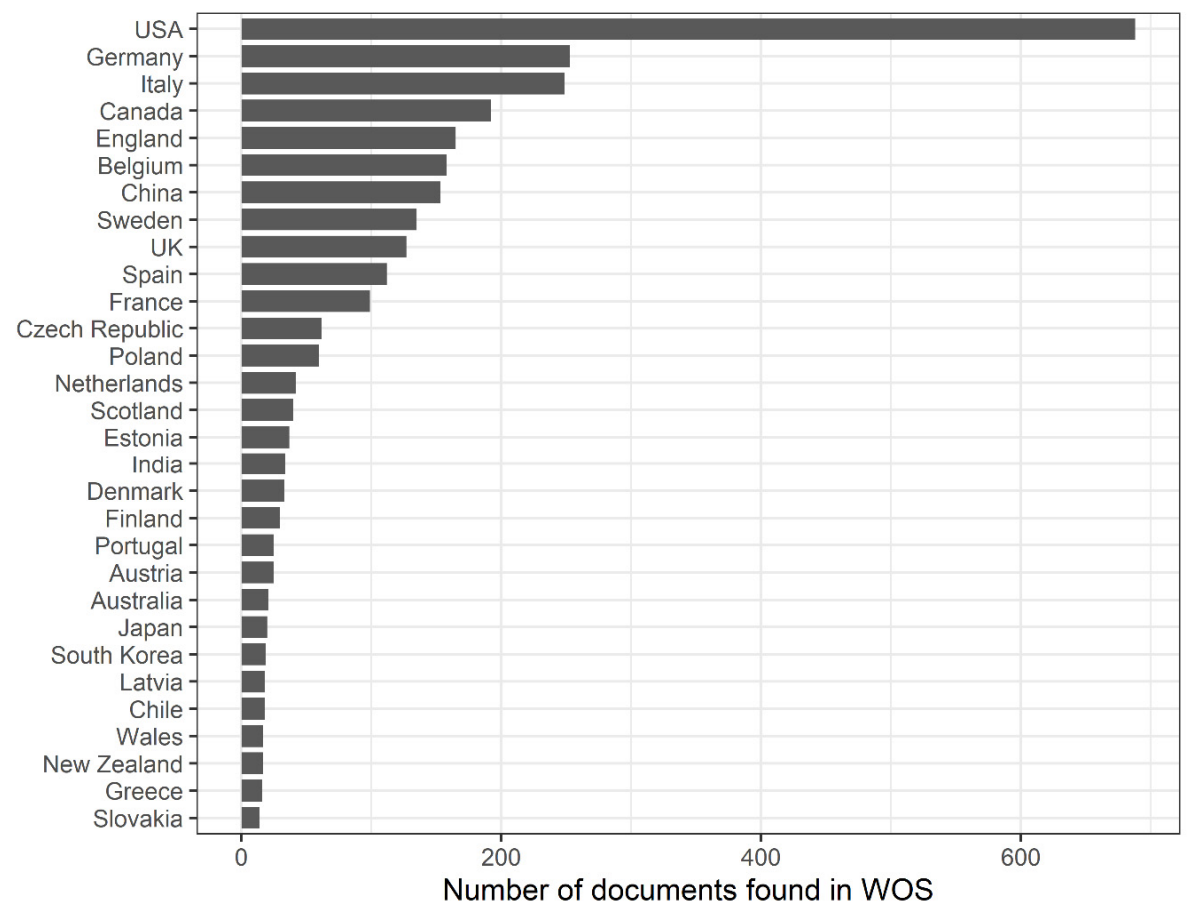

Figure 3. Ranking of the number of publications per country (including only the top 30 countries) found in the Web of Science (WOS) journal database, for the combination of the terms Populus or poplar, and short rotation or energy crop, in July 2020.

A classification of all the information available at a national level was performed according to the six categories detailed in Figure 2. This information was also broken down according to the availability of literature, separating the so-called gray literature from that contained in science journals and books (Figure 4). In Spain, biomass characterization and sustainability (mainly energy, economic, and environmental analysis) are the lines of research which have been explored the most based on scientific publications, although, if we include gray bibliography, then the most explored lines are production, modeling, and genetic material. Approximately $50.5 \%$ of the information evaluated corresponds to gray literature, $4.5 \%$ to books, and the rest to scientific publications (45\%).

\subsection{Suitability of Genetic Material}

\section{Clonal Selection}

Poplar is the model tree for genetic studies and is the furthest ahead in terms of biological knowledge and genetic resources [58]. Using the best adapted material when developing plantations helps to ensure the efficient use of site resources and therefore higher levels of production. Phenotypic plasticity refers to the capacity of an organism to alter its characteristics in response to environmental conditions [85]. The genotype by environment interaction $\left(G^{*} \mathrm{E}\right)$ is evidenced by the instability of phenotypic correlations derived from drastic differences in biomass production found in poplar plantations [86-88].

The $G^{*} E$ interaction makes poplar clonal recommendations more difficult, although it can also provide an opportunity to maximize production in these plantations by matching the most suitable material to specific site conditions. Genotypic stability is understood as the capacity of a cultivar to produce in accordance with the productive potential of each environment, that is, without straying from the behavior expected for the average genotypic value [89]. However, the strategies may differ, and this can be observed in different poplar SRC plantations; these strategies include attempting to find a broad adaptation or optimizing the adaptation to specific site conditions, hence the necessity to characterize the material [90-92]. This characterization not only has an impact on crop management, 
but also has repercussions for improvement strategies. In Spain, poplar material destined for biomass production has been characterized in recent years through the analysis of this interaction $[55,77,93-95]$.

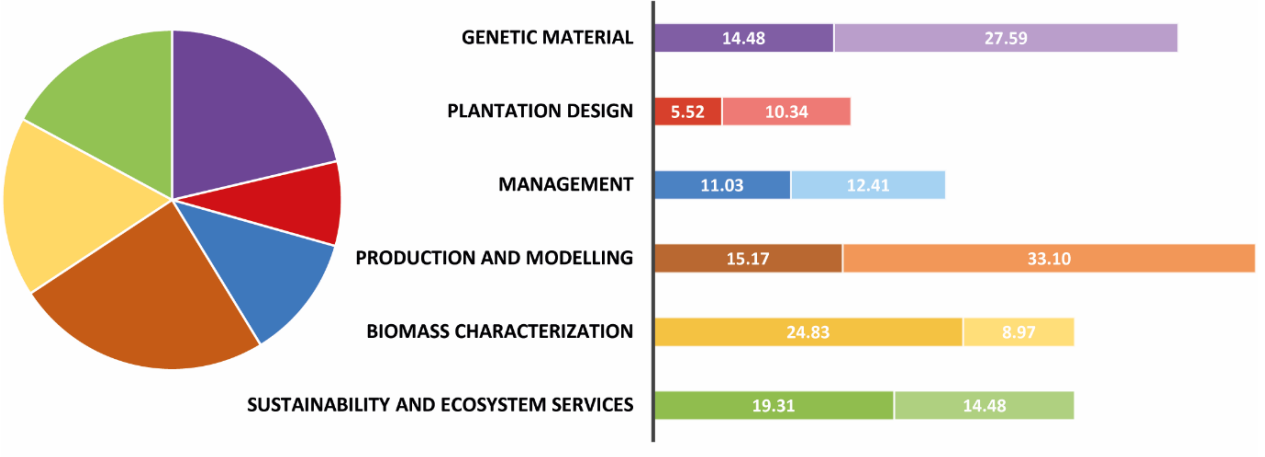

Figure 4. Percentages of Spanish publications in each of the six categories established. The light color corresponds to the gray literature on each subject.

With regards to the biomass, the development of genotypes aimed at achieving increased production, a greater tolerance to pests and diseases, and specific feedstock properties is ongoing through breeding programs in the countries spearheading the research, with the most prominent of these being the USA, Canada, Italy, France, Germany, and Sweden [58].

Traditional approaches based on recurrent selection as a way to increase hybrid vigor $[54,96]$ are now supported by genomics and phenomic techniques which contribute to accelerating these processes [97,98]. For example, tools such as genome editing using Crispr/Cas9 technology [99-101], the development of transgenic plants [102], complete genome association analysis [103], and new phenotypic tools [104], are now leading to important advances in genetic improvement of the Populus genus. A relevant review of the progress towards an improved biomass quality and resilience of production using both traditional approaches and new technology is provided by Clifton-Brown et al. [58], specifying the breeding programs in which each of these techniques has been used. New avenues of research have also been opened up through considering epigenetic inheritance, also termed soft inheritance. This relates to inheritable changes to the gene expression induced by environmental changes [105,106].

In Spain, although progress in this line of research is limited by the lack of specific breeding programs, progress has been made in the development and testing of different transformed poplar lines expressing a pine glutamine synthetase (GS) transgene involved in N assimilation [107]; the CsDML gene that induces bud formation needed for the survival of the apical meristem under the harsh conditions of winter [108]; and a gene related to ABI3/VP1 1 (CsRAV1) to increase syllepsis, and consequently biomass production, in commercial elite trees $[109,110]$. The biomass potential of the different available genotypes has also been tested, with multiple clonal trials involving traditional genotypes [95,111-116], as well as new genotypes specifically selected for biomass [77,117]. The results have revealed hybrids like 'Orion' and 'AF2' as site-specific genotypes, while 'I-214' and 'Monviso' have been demonstrated to adapt well to a wide variety of scenarios. Likewise, the ability of different genotypes to produce biomass in areas degraded by mining is also being tested, with some genotypes, such as 'Raspalje' and 'AF2', displaying acceptable yields [118]. In general, a strong correlation has been identified between the response to environmental variables and the taxonomic background, with the euramerican genotypes such as '2000 Verde' responding better to lower latitudes, higher mean temperatures of the vegetative period, and a lower sandy soil content, unlike interamerican genotypes such as 'Unal' [93].

\subsection{Plantation Design}

The design of a plantation is also a highly influential factor in stand management and the optimization of production [119]. Many different types of design have been tested in Spain, combining 
different plantation densities [120], tree distributions [121], and rotation lengths [122]. The possibility of mixing different species or different varieties of the same species has also been put forward as a way to optimize production.

\subsubsection{Densities}

A broad range of initial stocking rates have been employed to define the optimum plantation density, from 1000 cuttings ha ${ }^{-1}$ up to more than 40,000 cuttings ha ${ }^{-1}[123,124]$. Even higher densities have been contemplated, albeit less frequently, as in the case of DeBell et al. [125]. In Spain, a wide range of densities in multiple environments have been tested $[46,126,127]$. In terms of the yield, densities of around 20,000-25,000 cuttings ha ${ }^{-1}$ were the most productive in the first year, but the effect of competition reduced the differences between these and less stocked plantations as rotation approached three years [120]. Other factors, such as the costs associated with the higher densities due to the greater number of cuttings, increased demand for water, and increased consumption of consumables and time required for operations such as irrigation or harvesting [128], along with impediments to mechanization of the plantation, mean that densities in excess of 15,000 cuttings ha ${ }^{-1}$ are not recommended [120].

Furthermore, the position of the stools for a given density is also a matter of debate. Therefore, it is common for trials to include both single-row layouts [78,92] and double rows [13,129]. No significant differences in yield were found between the two layouts [130], although single-row designs tend to facilitate management operations. In Spain, both layouts have been tested [77,131], although a single row is more commonly used.

The density and distribution of stools should be directly linked to the rotation lengths applied, along with the maintenance and harvesting processes, always attempting to keep the production cost per biomass unit obtained as low as possible.

\subsubsection{Rotation Lengths and Number of Coppices}

The rotation is a factor closely linked to the density, as well as being influenced by the environment. Different rotations have been employed in the management of poplar SRC, from yearly to longer rotations for other productive objectives [132-135]. In recent years, rotations of 2 to 4 years have been the most frequently employed, with the final harvesting age depending on the genotype and site conditions $[90,129,136]$.

In Spain, rotations from 2 to 9 years have been tested [110,117,128,137], although few studies have compared different rotation lengths. Hernández Garasa et al. [138] determined that the maximum volume production occurred between 3 and 4 years, depending on the genotype planted. The suitability of a 3- or 4-year rotation length for different genotypes under poor site conditions was evaluated by Oliveira et al. [139], who noted that biomass production increases in most genotypes from $74 \%$ to $166 \%$ when the rotation length is extended by 1 year. However, some genotypes, such as 'AF2' and 'Dorskamp', presented no significant differences. These results are consistent with those obtained under average site conditions, where the maximum volume production was not reached with a 3-year rotation length, except in the cases of certain genotypes, such as 'A2A', 'A4A', and 'Lombardo Leones' [140]. Therefore, the rotation length chosen clearly depends on both the genotype and the site conditions, with longer rotation lengths being advisable when the conditions are not optimal. Further studies are needed in order to optimize the rotation length for specific sites, genotypes, and management conditions.

Another question that has sparked controversy is whether or not to coppice after the first establishment year to encourage multiple shoot growth [141,142]. This is a common practice in some countries, such as England [143]. In Spain, the use of management techniques both with or without coppicing after the first establishment year has also been studied [144-146], although no apparent advantage was found with regard to the additional coppice after the first establishment year, either in terms of biomass yield or quality. 
Opinions also vary regarding how many times coppicing should take place $[129,135]$, given that the lifecycle is clearly related not only to the rotation length, but also to the genotype and its interaction with the environment and the cultural practices applied, with all of these factors conditioning the useful life of the stool [68]. However, studies such as Štochlová et al. [147] suggest that five or six 3-year rotations are possible, although only one commercial clone-an interspecific hybrid of P. maximowiczii Rupr. $\times$ P. trichocarpa Torr \& A. Gray-was capable of biomass productivity compatible with the economic feasibility of cultivation. Therefore, it is important to take into account the factors limiting the duration of SRC crops. In Spain, several studies carried out over three successive rotations of 3 years each point to a decrease in biomass production during the third rotation cycle, suggesting the end of its productive cycle $[148,149]$. However, not all genotypes show the same behavior, with the genotypes 'I-214' and 'Monviso' presenting the highest yields during the third rotation [150].

\subsubsection{Species Mixtures}

There has been a sharp rise of interest in mixed forest plantations over recent decades because of the perceived benefits, not only for the environment and ecology, but also, although not always, for the yield, as a result of resource-use efficiency and facilitation. Although applying these designs in SRC plantations has attracted interest, there is still little information about it. Few examples exist of mixed plantations of poplar with other species in SRC. Some are mixtures of Populus spp. with Alnus spp. [151,152], the euramerican genotype 'AF2' with Ulmus spp. and Platanus $\times$ hispanica [153], and Populus spp. with Robinia spp. [154-156]. The mixture of Populus alba L.-Robinia pseudoacacia in SRC under Mediterranean conditions showed encouraging results in the first rotation [76], but they were not as promising in the second [157]. A similar design mixing R. pseudoacacia with the euramerican genotype 'Dorskamp' in central France demonstrated interspecific competition in the mixture as the preponderant interaction, resulting in higher mortality and lower biomass production than the two monocultures [155]. However, this mixture would appear to be advantageous given the different strategies shown by the two species in terms of the amount of litter and the dynamics of the main nutrients [158]. Therefore, although mixing the species does not increase the biomass yield, it may provide a good strategy for reducing future requirements for nitrogen addition (with the consequent ecological and economic implications), given the differences between the two monocultures in terms of processing the main nutrients [158]. Furthermore, both species-P. alba and R. pseudoacacia—are considered to be relatively tolerant to possible drought scenarios [111,159].

In any case, the current plantations are only experimental and their implementation on a commercial scale would involve restrictions in the spatial distribution as a result of the complex establishment and the harvesting requirements [76]. These difficulties often lead to mixing the species in lines or groups, thus losing the facilitation effect, although other benefits, such as the reduction of biotic and abiotic damage, landscape effects, and other environmental benefits, are retained.

Apart from mixtures of different species, complementarity is also explored in mixtures of varieties within the same genus [160,161]. Some examples in this regard include plurivarietal plantations of $P . \times$ generosa Henry [162], other hybrid groups of Populus [163], and different varieties of Salix spp. $[164,165]$, where the aim is to attain a greater tolerance to biotic and abiotic stresses while also increasing productivity.

\subsection{Management: Cultural Treatments}

There are many aspects of plantation management which have an impact on production.

\subsubsection{Control of Competing Vegetation}

Competition from weeds in the initial crop establishment stage is one of the main reasons for plantation failure. It is not only competition for water and nutrients, but also for light and space, which is crucial in the establishment stage. Deficits of these requirements can render the plantation unviable [68]. Therefore, weed control is considered a necessary practice, with treatments also being 
necessary during the first establishment year and after each coppicing. These treatments may involve both chemical and/or mechanical techniques [166-168].

Currently, the application of specific herbicides for poplar cultivation is very limited due to the European regulation on the sustainable use of pesticides and their commercialization (Directive 2009/128/CE of the European parliament; Regulation (CE) $n^{\circ} 1107 / 2009$ of the European parliament). In Spain, only six formulations are registered for use with hardwoods (RD 971/2014), but only one of them is specific for Populus.

\subsubsection{Fertilization}

The use of fertilizer in SRC plantations is a subject which attracts debate. There are many examples where fertilization provides no benefits $[59,90,169-171]$ and others where there are positive effects on production [145,172-177].

The lack of response to fertilization may be due to the fact that soil fertility is optimal or to an inadequate assessment of the limiting nutrients. The high $\mathrm{N}$ requirements of poplar $[178,179]$ mean that in locations where the soil is poor in organic matter, it is beneficial to apply fertilizer to increase the yield [180]. However, the excessive use of nitrogen fertilizers as part of conventional practice is increasingly being questioned because of environmental risks [181-183] and the high economic costs. Therefore, the use of alternatives to improve the nutritional status, such as designs with mixed plantations that include nitrogen fixing species [155] or alternative fertilization through the use of sewage sludge or waste water, have gained prominence in recent years [184-186]. Besides not having to use traditional fertilizers, these materials are purified and/or reused and the plantations thus act as phytoremediators [187]. All these alternative fertilization techniques have also been tested in poplar SRC plantations in Spain in recent years, although all in experimental plantations $[42,188,189]$.

In all cases, it is recommended that soil analysis be conducted prior to planting in order to optimize the use, where required, of fertilizers [190].

\subsubsection{Control of Pests and Diseases}

Newly emerging pests and diseases are one of the main problems facing agriculture in the 21st century due to the presence of extreme climatic conditions [191]. In poplar plantations, this risk has increased sharply as a result of the expansion of monoclonal plantations, with only a small number of different genotypes planted. There has been clear progress made in this area and it continues to be one of the main objectives in the breeding programs of the genus.

Examples of the most serious pests and diseases affecting the leaves are those caused by Melampsora spp. (rusts), Marssonina brunnea (Ell. et Ev.) Magn., Phloeomyzus passerinii Sign. (woolly poplar aphid), Venturia populina (Vuill.) Fabr., Chrysomela populi L. (red poplar leaf beetle), and Leucoma salicis L. (white satin moth), whilst those affecting the stems and trunks are Mycosphaerella populorum Peck (stem canker), Cryptorhynchus lapathi L. (poplar and willow borer), Paranthrene tabaniformis Rott. (dusky clearwing), and Sesia apiformis L., among others, although these are not as relevant to the SRC crop [192-197].

In Spain, although phytosanitary problems in SRC crops have not occurred very frequently, the presence of rust has been observed in rust-prone genotypes when grown for timber production. Chrysomela populi L. has been detected in plantations in the northern half of the country, as well as Corythucha ciliata (Say) in the center of the peninsula, necessitating the timely application of phytosanitary products.

In any case, the sustainability of these plantations depends on the use of genetic material which is tolerant or resistant to these types of stress [68,198], as well as resorting to biological control in the case of certain pests. 


\subsubsection{Irrigation}

Given the marked hygrophilic nature of the Populus genus [199], the availability of water in the soil is one of the limiting factors for its cultivation [200]. Due to the Mediterranean climatic conditions, it is necessary to irrigate SRC plantations in Spain during the summer drought season [201-203]. Due to limitations on water use at a global scale [204], the irrigation of SRC crops is viewed with caution in areas where this practice is necessary. This has led to changes in the way irrigation is applied, moving away from flood irrigation towards more efficient systems such as drip irrigation, although much more can still be done to increase the technical efficiency [205].

Water restrictions generally lead to production losses [206,207], although a high variability has been found in response to drought conditions. For this reason, the identification of genotypes with a greater water-use efficiency through different methodological approaches is undoubtedly of interest [208], seeking to combine materials with a high productivity and greater water-use efficiency [209]. This is especially true in the Mediterranean area [77,210-213], although accepting a certain loss of production may be advisable in these scenarios [205].

Highly productive genotypes such as 'AF2' and 'Monviso' have exhibited the greatest water-use efficiency under optimal conditions, although under restrictive water conditions, they have presented a similar water-use efficiency to that of the less productive genotypes. The strategy followed by all of them to improve the intrinsic water-use efficiency seems to be linked to stomatal control, rather than differences in the rate of photosynthesis [205].

In addition to the implications in terms of sustainability, the economic implications must also be taken into account, bearing in mind that irrigation is one of the limiting factors when assessing the profitability of these plantations under Mediterranean conditions. It has been calculated that the costs associated with irrigation, which include the irrigation system, maintenance, and the annual costs of water and electricity, account for $30 \%$ of the total costs over a whole cycle of 12 years [214].

The reusing of water from different sources represents a new approach in the context of SRC $[187,188]$, with a solid background in the past $[215,216]$. In any case, it is necessary to increase the amount of research into the breeding of plants for production under conditions of water scarcity at a global level, especially in regions that suffer from water restrictions.

These alternatives, together with the improvement in irrigation techniques, could provide solutions to ensure that viable production is attained in areas where water use must be minimized, such as in Mediterranean environments.

\subsection{Production}

Short rotation coppice plantations (SRC) provide a viable alternative for the production of quality lignocellulosic biomass [217]. The biomass produced in SRC is characterized by a predictable periodicity and yield, depending on the area of cultivation. The main challenge with regard to these crops is to achieve a high level of sustainable production while maximizing benefits; that is, combining economic viability with environmental sustainability.

According to a review by Sixto et al. [68] concerning biomass production in this type of plantation using poplar genotypes, a large quantity of literature exists on the different clonal productivity under a range of environments. Table 2 presents some examples of biomass production obtained in different European countries, ranging widely from 1 to $24 \mathrm{Mg} \mathrm{dm}$ (dry matter) ha ${ }^{-1}$ year $^{-1}$, depending on the site characteristics, the genetic material, the design, and the management scheme. Table 3 presents the production obtained in Spain under Mediterranean conditions, ranging widely from 1 to $37 \mathrm{Mg} \mathrm{dm} \mathrm{ha}^{-1}$ year $^{-1}$. The largest reported productions appear to be those associated with Mediterranean irrigated environments. Despite this, the average potential production at a national level in Spain is estimated to be around $15.3 \mathrm{Mg} \mathrm{dm} \mathrm{ha}^{-1}$ year $^{-1}$ for plantations with standard management schemes [47], although there is also high variability, depending on the previously mentioned factors $[46,55,77,145,218]$. 
Table 2. Different examples of the biomass yield $\left(\mathrm{Mg} \mathrm{dm} \mathrm{ha}^{-1}\right.$ year ${ }^{-1}$ ) per genotype, age, and rotation in poplar short rotation plantations in Europe (excluding Spain).

\begin{tabular}{|c|c|c|c|c|c|c|c|c|}
\hline \multirow{2}{*}{ Species and Poplar Hybrids } & \multirow{2}{*}{ Genotype } & \multirow{2}{*}{ Density } & \multirow{2}{*}{ Age } & \multirow{2}{*}{ Country } & \multicolumn{3}{|c|}{ Yield (Mg dm ha ${ }^{-1}$ Year $^{-1}$ ) by Rotation } & \multirow{2}{*}{ Reference } \\
\hline & & & & & 1st & 2nd & 3rd & \\
\hline \multirow{2}{*}{$\begin{array}{c}\text { P. deltoides } \times \text { P. trichocarpa } \\
\text { P. nigra }\end{array}$} & 'IBW2' & & & & 2.5 & 1.6 & & \\
\hline & 'Wolterson' & & & & 8.1 & 9.7 & & \\
\hline \multirow[t]{2}{*}{ P. trichocarpa } & 'Fritzi Pauley' & 10000 & & Belgium & 8.1 & 8.2 & & [19 219] \\
\hline & 'Trichobel' & 10,000 & 4 & Deigium & 8.4 & 8.2 & & {$[19,219]$} \\
\hline P. $\times$ canadensis & 'Gibecq' & & & & 1.6 & 3.5 & & \\
\hline P. $\times$ generosa & 'Hazendans' & & & & 10.8 & 3 & & \\
\hline \multirow{3}{*}{$\begin{array}{c}\text { P. deltoides } \times P . \times \text { generosa } \\
\text { P. nigra } \\
\text { P. nigra } \times \text { P. maximowiczii }\end{array}$} & 'Grimminge' & & & & 4.3 & 11.7 & 8.4 & \\
\hline & 'Brandaris' & & & & 1.4 & 7.0 & 8.7 & \\
\hline & 'Wolterson' & & & & 2.7 & 11.7 & 14.6 & \\
\hline \multirow{5}{*}{ P. trichocarpa $\times$ P. maximowiczii } & 'Bakan' & & & & 4.9 & 14.3 & 18.1 & \\
\hline & 'Skado' & & & & 5.7 & 16.8 & 20.8 & \\
\hline & 'Ellert' & & 2 (In the 3rd rot & & 3.3 & 11.1 & 19.5 & \\
\hline & 'Hees' & 8000 & the age is 3 year) & Belgium & 6.5 & 15.5 & 26.0 & {$[136]^{*}$} \\
\hline & 'Koster' & & & & 2.8 & 9.5 & 14.1 & \\
\hline \multirow{2}{*}{ P. $\times$ canadensis } & 'Robusta' & & & & 2.5 & 8.1 & 15.6 & \\
\hline & 'Vesten & & & & 4.7 & 12.6 & 16.1 & \\
\hline P. maximowiczii $\times$ P. trichocarpa & 'NE-42' & 2222 & 3 (2nd rot: 4 year) & Czech Republic & $\begin{array}{c}1.0-1.4 \\
8.3\end{array}$ & $\begin{array}{c}9.4-9.8 \\
15.4\end{array}$ & $\begin{array}{c}9.1-11.4 \\
189\end{array}$ & {$[220]$} \\
\hline
\end{tabular}


Table 2. Cont

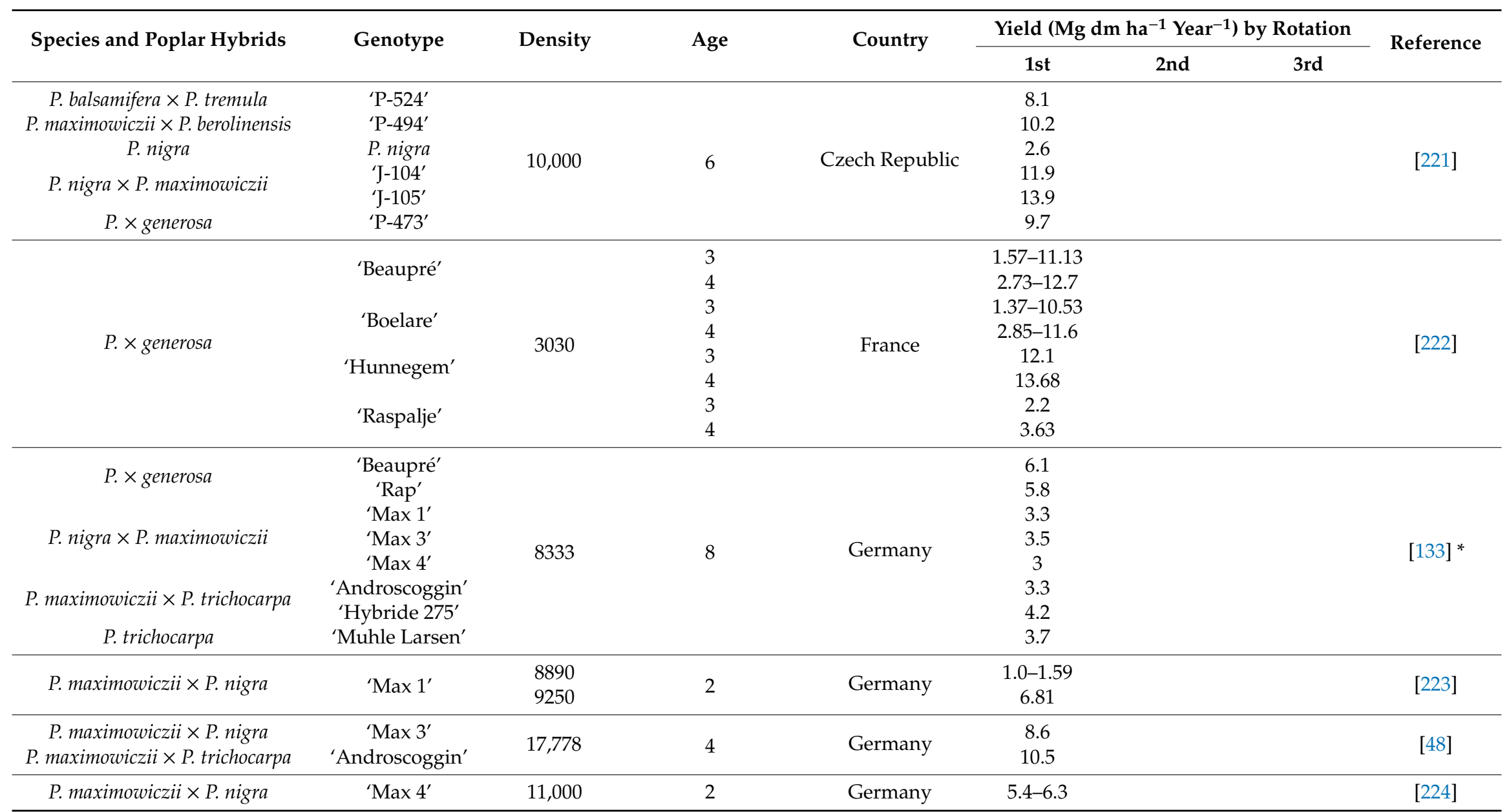


Table 2. Cont

\begin{tabular}{|c|c|c|c|c|c|c|c|c|}
\hline \multirow{2}{*}{ Species and Poplar Hybrids } & \multirow{2}{*}{ Genotype } & \multirow{2}{*}{ Density } & \multirow{2}{*}{ Age } & \multirow{2}{*}{ Country } & \multicolumn{3}{|c|}{ Yield (Mg dm ha-1 Year $^{-1}$ ) by Rotation } & \multirow{2}{*}{ Reference } \\
\hline & & & & & 1st & 2nd & 3rd & \\
\hline \multirow{7}{*}{ P. deltoides } & \multirow{2}{*}{ 'Baldo' } & \multirow{2}{*}{$\begin{array}{c}5747 \\
10,000\end{array}$} & \multirow{15}{*}{2} & \multirow{15}{*}{ Italy } & 4.75 & & & \\
\hline & & & & & 17.5 & & & \\
\hline & 'Dvina' & 10,000 & & & 11.8 & & & \\
\hline & 'Lambro' & 10,000 & & & 9.5 & & & \\
\hline & 'Lena' & 10,000 & & & 14.2 & & & \\
\hline & & 5714 & & & 7.05 & & & \\
\hline & ‘Lux’ & 8333 & & & 4.3 & & & \\
\hline \multirow{8}{*}{ P. $\times$ canadensis } & \multirow{2}{*}{ 'BL-Costanzo' } & 5714 & & & 3.25 & & & {$[90]$} \\
\hline & & 7142 & & & 4.45 & & & \\
\hline & \multirow{2}{*}{ ‘Cima' } & 5714 & & & 4.55 & & & \\
\hline & & 7142 & & & 5.7 & & & \\
\hline & \multirow{2}{*}{ 'I-214' } & 5747 & & & 4.35 & & & \\
\hline & & 10,000 & & & 8.4 & & & \\
\hline & \multirow{2}{*}{ 'Luisa Avanzo' } & 5714 & & & 5.55 & & & \\
\hline & & 7142 & & & 6.25 & & & \\
\hline \multirow{2}{*}{ P. deltoides } & \multirow{2}{*}{ 'Lux' } & \multirow{2}{*}{10,000} & 2 & \multirow{2}{*}{ Italy } & \multirow[t]{2}{*}{12.38} & 6.99 & 4.99 & \multirow{2}{*}{ [225] } \\
\hline & & & 3 & & & 7.07 & 14.53 & \\
\hline \multirow{3}{*}{ P. deltoides } & \multirow{3}{*}{ ‘Lux' } & \multirow{3}{*}{10,000} & 1 & \multirow{3}{*}{ Italy } & & & 16.4 & \\
\hline & & & 2 & & 22.5 & & & [135] \\
\hline & & & 3 & & 24.3 & & & \\
\hline P. deltoides & 'Lux' & & & & $3.31-9.33$ & $2.17-18.85$ & & \\
\hline P. maximowiczii $\times$ P. nigra & 'AF10' & & & & 14.84 & 20.74 & & \\
\hline & 'AF2' & & & & $5.66-15.77$ & $7.54-17.01$ & & \\
\hline P. $\times$ canadensis & 'I-214' & & & Italy & $3.63-11.87$ & $4.2-18.44$ & & [226] \\
\hline & 'Sirio' & 5900 & 2 & Italy & 5.65 & 8.55 & & [226] \\
\hline$P \times$ oenerosa $\times P$ niora & 'AF6' & & & & $5.76-15.04$ & $5.79-17.07$ & & \\
\hline P. $\times$ generosa $\times$ P. nigra & 'Monviso' & & & & $6.79-17.92$ & $9.31-23.55$ & & \\
\hline P. $\times$ generosa $\times$ P. trichocarpa & 'AF8' & & & & $4.75-14.93$ & $5.04-24.05$ & & \\
\hline
\end{tabular}


Table 2. Cont

\begin{tabular}{|c|c|c|c|c|c|c|c|c|}
\hline \multirow{2}{*}{ Species and Poplar Hybrids } & \multirow{2}{*}{ Genotype } & \multirow{2}{*}{ Density } & \multirow{2}{*}{ Age } & \multirow{2}{*}{ Country } & \multicolumn{3}{|c|}{ Yield (Mg dm ha-1 Year- $^{-1}$ ) by Rotation } & \multirow{2}{*}{ Reference } \\
\hline & & & & & 1 st & 2nd & 3 rd & \\
\hline \multirow{2}{*}{ P. $\times$ canadensis } & 'AF2' & & & & 16 & 17 & 17 & \\
\hline & 'I-214' & & & & 11.5 & 18 & 13.5 & \\
\hline \multirow{2}{*}{ P. $\times$ generosa $\times$ P. nigra } & 'AF6' & 6061 & 2 & Italy & 15 & 17.5 & 15 & {$[92] *$} \\
\hline & 'Monviso' & & & & 17.5 & 23.5 & 17.5 & \\
\hline P. $\times$ generosa $\times P$. trichocarpa & 'AF8' & & & & 15 & 24 & 19 & \\
\hline P. maximowiczii $\times$ P. trichocarpa & 'NE-42' & & & & 8 & & & \\
\hline \multirow{3}{*}{ P. trichocarpa $\times$ P. trichocarpa } & 'Fritzi Pauley' & & & & 8.1 & & & \\
\hline & 'AF2' & & & & 4.1 & & & \\
\hline & 'Albelo' & & & & 4 & & & \\
\hline \multirow[t]{3}{*}{ P. $\times$ canadensis } & 'Degrosso' & 1333 & 7 & Poland & 6.8 & & & {$[52]$} \\
\hline & 'Koster' & & & & 5.6 & & & \\
\hline & 'Polargo' & & & & 4.3 & & & \\
\hline \multirow{2}{*}{$\begin{array}{l}\text { P. } \times \text { generosa } \times \text { P. trichocarpa } \\
\text { P. nigra } \times \text { P. maximowiczii }\end{array}$} & 'AF8' & & & & 2.5 & & & \\
\hline & $' \operatorname{Max} 5^{\prime}$ & 11,110 & 3 & Poland & 7.8 & & & [227] \\
\hline \multirow[b]{2}{*}{ P. trichocarpa } & 'Columbia River' & & & & 6.71 & 6.62 & & \\
\hline & 'Fritzi Pauley' & & & & 8.59 & 8.24 & & \\
\hline \multirow[t]{2}{*}{ P. trichocarpa $\times P$. balsamifera } & ‘Balsam Spire' & & & & 7.24 & 7.03 & & \\
\hline & 'Gaver' & & & & 6.58 & 5.58 & & \\
\hline \multirow[t]{2}{*}{ P. $\times$ canadensis } & 'Ghoy' & & & & 6.45 & 5.77 & & \\
\hline & 'Gibecq' & 10,000 & 3 & $\begin{array}{l}\text { United } \\
\text { Kingdom }\end{array}$ & 5.7 & 4.73 & & [13] \\
\hline \multirow{6}{*}{ P. $\times$ generosa } & 'Beaupré' & & & Kingdom & 7.34 & 4.87 & & \\
\hline & 'Boelare' & & & & 6.23 & 4.2 & & \\
\hline & 'Hazendans' & & & & 7.23 & 7.56 & & \\
\hline & 'Hoogvorst' & & & & 8.84 & 8.12 & & \\
\hline & 'Raspalje' & & & & 6.69 & 4.66 & & \\
\hline & 'Unal' & & & & 7.55 & 5.25 & & \\
\hline \multirow{3}{*}{ P. balsamifera $\times P$. trichocarpa } & & 10,000 & 5 & & 14.42 & & & \\
\hline & 'Balsam Spire' & 4444 & 5 & $\begin{array}{l}\text { United } \\
\text { Kingdom }\end{array}$ & 11.20 & & & [228] \\
\hline & & 10,000 & 4 & Kingdom & 13.98 & & & \\
\hline
\end{tabular}

* Estimated values from graphs. 
Table 3. Different examples of the biomass yield $\left(\mathrm{Mg} \mathrm{dm} \mathrm{ha}^{-1}\right.$ year $\left.^{-1}\right)$ per genotype, age, and rotation in poplar short rotation plantations in Spain under Mediterranean conditions.

\begin{tabular}{|c|c|c|c|c|c|c|c|c|}
\hline \multirow{2}{*}{ Species and Poplar Hybrids } & \multirow{2}{*}{ Genotype } & \multirow{2}{*}{ Density } & \multirow{2}{*}{ Age } & \multirow{2}{*}{ Location } & \multicolumn{3}{|c|}{ Yield $\left(\mathrm{Mg} \mathrm{dm} \mathrm{ha} \mathrm{h}^{-1}\right.$ Year-1) by Rotation $^{-1}$} & \multirow{2}{*}{ Reference } \\
\hline & & & & & 1 st & 2nd & 3rd & \\
\hline \multirow{3}{*}{ P. $\times$ canadensis } & 'AF2' & \multirow{4}{*}{13,333} & \multirow{4}{*}{3} & \multirow{4}{*}{ Aragón } & 24.72 & & & \multirow{4}{*}[229]{$*$} \\
\hline & 'Ballottino' & & & & 25.08 & & & \\
\hline & 'I-214' & & & & 28.64 & & & \\
\hline P. $\times$ generosa $\times P$. nigra & 'Monviso' & & & & 26.91 & & & \\
\hline \multirow{4}{*}{$\begin{array}{l}\text { P. } \times \text { canadensis } \\
P . \times \text { generosa }\end{array}$} & 'Campeador' & \multirow{4}{*}{10,000} & \multirow{4}{*}{5} & \multirow{4}{*}{ Soria } & 12.10 & & & \multirow{4}{*}{ [230] } \\
\hline & 'Dorskamp' & & & & 6.3 & & & \\
\hline & 'Boelare' & & & & 16.6 & & & \\
\hline & 'Raspalje' & & & & 19.8 & & & \\
\hline \multirow{3}{*}{ P. $\times$ canadensis } & 'Campeador' & \multirow{3}{*}{10,000} & \multirow[b]{3}{*}{6} & \multirow{9}{*}{ Soria } & 9.3 & \multirow{9}{*}{17.2} & \multirow{9}{*}{14.8} & \multirow{9}{*}[126,127]{} \\
\hline & 'I-214' & & & & 9.9 & & & \\
\hline & 'Boelare' & & & & 12.9 & & & \\
\hline \multirow{6}{*}{ P. $\times$ generosa } & \multirow{6}{*}{ 'Raspalje' } & 2500 & \multirow{4}{*}{6 (2nd-3rd:5year) } & & 8.0 & & & \\
\hline & & 5000 & & & 9.2 & & & \\
\hline & & 10,000 & & & 16.5 & & & \\
\hline & & 10,000 & & & 9.8 & & & \\
\hline & & 20,000 & 3 & & 12.7 & & & \\
\hline & & 40,000 & & & 15.6 & & & \\
\hline \multirow{2}{*}{ P. $\times$ canadensis } & 'AF2' & \multirow{8}{*}{8000} & \multirow{8}{*}{7} & & 0.84 & & & \\
\hline & 'I-214' & & & & 2.96 & & & \\
\hline & 'Beaupré' & & & & 3.07 & & & [231] Without irrigation \\
\hline P. $\times$ generosa & 'Raspalje' & & & & 3.1 & & & in a transition zone \\
\hline & 'Unal' & & & Lugo & 1.97 & & & between Mediterranean \\
\hline & 'AF6' & & & & 1.14 & & & and Atlantic climate \\
\hline P. $\times$ generosa $\times$ P. negra & 'Monviso' & & & & 0.93 & & & \\
\hline P. trichocarpa & 'Trichocarpa' & & & & 3.93 & & & \\
\hline P. $\times$ canadensis & 'AF2' and 'I-214' & 33,333 & 3 & Soria & $7-12$ & $2-9.5$ & $1.5-4.5$ & {$[145]^{*}$} \\
\hline P. $\times$ canadensis & 'I-214' & 10,000 & 3 & Asturias & 9 & 11 & 6 & $\begin{array}{c}{[148] \text { * Without }} \\
\text { irrigation in mining zone }\end{array}$ \\
\hline P. $\times$ canadensis & 'I-214' & 37,037 & 2 & Salamanca & 12.41 & 9.99 & 24.83 & [232] \\
\hline
\end{tabular}


Table 3. Cont.

\begin{tabular}{|c|c|c|c|c|c|c|c|c|}
\hline \multirow{2}{*}{ Species and Poplar Hybrids } & \multirow{2}{*}{ Genotype } & \multirow{2}{*}{ Density } & \multirow{2}{*}{ Age } & \multirow{2}{*}{ Location } & \multicolumn{3}{|c|}{ Yield (Mg dm ha ${ }^{-1}$ Year $^{-1}$ ) by Rotation } & \multirow{2}{*}{ Reference } \\
\hline & & & & & 1 st & 2nd & 3rd & \\
\hline & 'AF2' & \multirow{6}{*}{13,333} & \multirow{6}{*}{3} & \multirow{6}{*}{ Aragón } & 15.53 & & & \multirow{6}{*}[233]{$^{*},[234]$} \\
\hline P. $\times$ canadensis & 'I-214' & & & & 10.3 & & & \\
\hline & 'Guardi' & & & & 9.03 & & & \\
\hline P. deltoides & 'Viriato' & & & & 14 & & & \\
\hline$P . \times$ generosa & 'Unal' & & & & 9.23 & & & \\
\hline P. $\times$ generosa $\times$ P. nigra & 'Monviso' & & & & 13.13 & & & \\
\hline P. $\times$ canadensis & 'I-214' & 33,333 & 2 & Spain & 15.1 & & & [112] \\
\hline Populus spp. & 4 genotypes & 13,333 & 3 & Granada & 13.7 & & & \\
\hline Populus spp. & 3 genotypes & 19,700 & 4 & Soria & 12.0 & & & \\
\hline Populus spp. & 4 genotypes & 13,333 & 3 & Zamora & 7.7 & & & [235] \\
\hline Populus spp. & 4 genotypes & 33,333 & 3 & León & 6.9 & & & \\
\hline Populus spp. & 4 genotypes & 20,000 & 3 & Navarra & 16 & & & \\
\hline \multirow{7}{*}{ P. $\times$ canadensis } & $\begin{array}{l}\text { 'Lombardo } \\
\text { leones' }\end{array}$ & \multirow{7}{*}{17,316} & \multirow{7}{*}{3} & \multirow{7}{*}{ Soria } & 9.1 & 17.1 & & \multirow{7}{*}{ [236] } \\
\hline & 'A4A' & & & & 20.0 & 12.2 & & \\
\hline & 'A $2 A^{\prime}$ & & & & 11.8 & 15.3 & & \\
\hline & 'I-214' & & & & 22.0 & 30.1 & & \\
\hline & 'Luisa Avanzo' & & & & 17.3 & 27.1 & & \\
\hline & 'MC' & & & & 16.8 & 18.4 & & \\
\hline & '2000 Verde' & & & & 13.64 & 14.25 & & \\
\hline \multirow{5}{*}{ P. $\times$ canadensis } & 'Baldo' & \multirow{5}{*}{10,000} & \multirow{5}{*}{3} & & $8.25-19.07$ & $8.59-29.94$ & & \multirow{5}{*}[77,80]{} \\
\hline & 'Ballottino' & & & Gerona & $8.25-15.94$ & $11.45-19.20$ & & \\
\hline & 'I-214' & & & Madrid, & $5.45-16.16$ & $11.09-37.27$ & & \\
\hline & 'Orion' & & & Soria & $12.53-22.65$ & $12.70-34.56$ & & \\
\hline & 'Oudenberg' & & & & $9.43-14.27$ & $9.66-19.96$ & & \\
\hline \multirow{12}{*}{ P. $\times$ canadensis } & \multirow{12}{*}{ 'I-214' } & \multirow{12}{*}{$6666-33,333$} & \multirow{12}{*}{3} & Barcelona & 4.2 & & & \multirow{12}{*}{ [47] } \\
\hline & & & & Gerona & 5.7 & & & \\
\hline & & & & Gerona & 11.6 & & & \\
\hline & & & & Granada & 18.3 & & & \\
\hline & & & & León & 4.7 & & & \\
\hline & & & & León & 5.8 & & & \\
\hline & & & & León & 15.2 & & & \\
\hline & & & & Madrid & 13.2 & & & \\
\hline & & & & Navarra & 18.5 & & & \\
\hline & & & & Soria & 8.1 & & & \\
\hline & & & & Soria & 14.2 & & & \\
\hline & & & & Zamora & 8.2 & & & \\
\hline
\end{tabular}


Table 3. Cont.

\begin{tabular}{|c|c|c|c|c|c|c|c|c|}
\hline \multirow{2}{*}{ Species and Poplar Hybrids } & \multirow{2}{*}{ Genotype } & \multirow{2}{*}{ Density } & \multirow{2}{*}{ Age } & \multirow{2}{*}{ Location } & \multicolumn{3}{|c|}{ Yield (Mg dm ha ${ }^{-1}$ Year $\left.^{-1}\right)$ by Rotation } & \multirow{2}{*}{ Reference } \\
\hline & & & & & 1st & 2nd & 3 rd & \\
\hline \multirow{7}{*}{ P. $\times$ canadensis } & 'I-214' & \multirow{6}{*}{5000} & & \multirow{7}{*}{ Madrid } & 12.4 & & & \multirow{7}{*}{ [44] } \\
\hline & 'Campeador' & & 4 & & 10.0 & & & \\
\hline & 'Canada Blanco' & & & & 8.4 & & & \\
\hline & 'I-214' & & 2 & & 10.90 & & & \\
\hline & 'I-214' & & 3 & & 10.10 & & & \\
\hline & 'I-214' & & 5 & & 17.30 & & & \\
\hline & 'I-214' & 10,000 & 4 & & 16.9 & & & \\
\hline P. $\times$ canadensis & 'AF2' & 13,333 & 3 & Granada & 17.1 & & & [237] \\
\hline \multirow{5}{*}{ P. $\times$ canadensis } & 'AF2' & \multirow{9}{*}{33,333} & \multirow{9}{*}{3} & \multirow{9}{*}{ León } & 16.75 & & & \multirow{9}{*}{ [122] } \\
\hline & 'Guardi' & & & & 16.14 & & & \\
\hline & 'I-214' & & & & 15.14 & & & \\
\hline & 'MC' & & & & 15.01 & & & \\
\hline & '2000 Verde' & & & & 10.05 & & & \\
\hline P. $\times$ generosa & 'Unal' & & & & 11.38 & & & \\
\hline 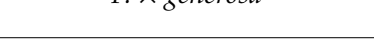 & 'USA 49-177' & & & & 15.92 & & & \\
\hline \multirow{2}{*}{ P. $\times$ generosa $\times$ P. nigra } & 'Monviso' & & & & 15.16 & & & \\
\hline & 'Pegaso' & & & & 7.37 & & & \\
\hline \multirow{5}{*}{ P. $\times$ canadensis } & 'AF2' & \multirow{8}{*}{33,333} & \multirow{8}{*}{3} & \multirow{8}{*}{$\begin{array}{l}\text { León, } \\
\text { Gerona, } \\
\text { Madrid, } \\
\text { Soria }\end{array}$} & 16.8 & & & \multirow{8}{*}{ [55] } \\
\hline & 'Guardi' & & & & 12.44 & & & \\
\hline & 'I-214' & & & & 12.21 & & & \\
\hline & 'MC' & & & & 15.32 & & & \\
\hline & ‘2000 Verde' & & & & 15.38 & & & \\
\hline$P . \times$ generosa & 'Unal' & & & & 11.13 & & & \\
\hline \multirow{2}{*}{$P . \times$ generosa $\times P$. nigra } & 'Monviso' & & & & 14.47 & & & \\
\hline & 'Pegaso' & & & & 6.65 & & & \\
\hline \multirow[t]{2}{*}{ P. deltoides } & 'Viriato' & & & & 9.55 & 18.20 & 12.82 & \\
\hline & ‘AF2’ & & & & 13.47 & 16.66 & 13.99 & \\
\hline \multirow[t]{2}{*}{ P. $\times$ canadensis } & 'Ballottino' & 13,333 & 3 & Granada & 9.48 & 16.75 & 12.27 & [150] \\
\hline & 'I-214' & & & & 9.11 & 13.44 & 15.81 & \\
\hline$P . \times$ generos $a \times P$. nigra & 'Monviso' & & & & 12.29 & 11.57 & 13.92 & \\
\hline
\end{tabular}


Table 3. Cont

\begin{tabular}{|c|c|c|c|c|c|c|c|c|}
\hline \multirow{2}{*}{ Species and Poplar Hybrids } & \multirow{2}{*}{ Genotype } & \multirow{2}{*}{ Density } & \multirow{2}{*}{ Age } & \multirow{2}{*}{ Location } & \multicolumn{3}{|c|}{ Yield (Mg dm ha ${ }^{-1}$ Year $^{-1}$ ) by Rotation } & \multirow{2}{*}{ Reference } \\
\hline & & & & & 1st & 2nd & 3rd & \\
\hline \multirow{6}{*}{ P. $\times$ canadensis } & 'AF2' & \multirow{11}{*}{20,000} & \multirow{11}{*}{3 (2nd rot: 2 year) } & \multirow{11}{*}{ Navarra } & 15.59 & & & \multirow{11}{*}{ [238] } \\
\hline & 'A4A' & & & & 23.80 & 22.12 & & \\
\hline & 'Guardi' & & & & 15.59 & 6.43 & & \\
\hline & 'I-214' & & & & 23.80 & 18.51 & & \\
\hline & ${ }^{\prime} \mathrm{MC}^{\prime}$ & & & & 11.21 & 26.51 & & \\
\hline & 'Triplo' & & & & 18.07 & 32.92 & & \\
\hline P. deltoides & 'Viriato' & & & & 16.68 & 38.18 & & \\
\hline & ‘Beaupré’ & & & & 21.29 & 15.29 & & \\
\hline P. $\times$ generosa & 'Unal' & & & & 25.86 & 12.15 & & \\
\hline \multirow{2}{*}{ P. $\times$ generosa $\times$ P. nigra } & 'Monviso' & & & & 15.41 & & & \\
\hline & 'Pegaso' & & & & 13.99 & & & \\
\hline \multirow{4}{*}{ P. $\times$ canadensis } & \multirow{4}{*}{ 'I-214' } & 15,000 & \multirow{4}{*}{3 (2nd rot:2 year) } & \multirow{4}{*}{ Navarra } & 18.20 & 14.46 & & \multirow{4}{*}{ [239] } \\
\hline & & 20,000 & & & 18.68 & 18.51 & & \\
\hline & & 25,000 & & & 23.36 & 14.65 & & \\
\hline & & 33,333 & & & 18.85 & 17.13 & & \\
\hline
\end{tabular}

* Estimated values from graphs. 
Modeling of Growth, Production, and Biometric Relationships in SRC Poplar Plantations

The use of estimation models to predict available biomass is becoming more frequent as research into this type of plantation progresses, not only because of the costs involved in carrying out direct estimates [240], but also due to the greater flexibility and the possibility of extrapolating the results to larger scales when the models are based on a wide range of empirical data.

Allometric models which relate the tree diameter or another easily measurable variable to the biomass are those most commonly used in forest inventories or ecological studies [241,242]. These models have also been employed in SRC plantations to estimate the available biomass [243-245]. These predictions are particularly important when evaluating the economic viability of a crop [47,246]. Apart from the choice of regression model, the assumptions that underlie the regression procedures, and the data transformations used during the procedures [247], we are confronted with a large number of factors which create uncertainty and may affect the final results [248]. Simple equations which are valid for a wide range of conditions and plant material are usually preferred. There are several examples used for poplar SRC plantations [18,30,78,136,171,226,249-253]. The precision of such allometric equations is generally sufficient for stem biomass components [254]. However, other examples of equations exist that include more predictive variables in the models, which can improve the precision of the estimations [11,47,205,255-258]. Although specific genotype-level models provide the greatest precision, in cases where genotype identification is complicated, models for genotypes which are taxonomically close or taxonomic-specific models are often used. However, under Mediterranean conditions at least, Oliveira et al. [252] proves that the genetic origin does not explain the similarities in biomass allometry among genotypes, so these approaches are not always advisable.

Over the last decade, considerable advances have been made with regards to modeling to estimate biomass from SRC poplar plantations. Not only have advances been made in allometric models, but also much effort has been channeled towards modeling other aspects of growth in these plantations, such as the leaf architecture [259], root production [260-262], and the use of process models with this aim [263-267], in addition to other management tools derived from models [47,268-270].

In recent years, considerable progress has been made in the development of specific predictive models for SRC plantations under Mediterranean conditions. Improvements in the model development methodology to achieve more robust biomass predictions have been made [248,271], and the suitability of biomass models for local populations, as well as their performance for different sample sizes, have also been evaluated [253]. Different models have been developed, including a dynamic, whole-stand model for 'I-214' poplar genotype plantations in the northern and central plateau in Spain [272], along with individual tree models and general models for estimating both the above- and below-ground biomass in poplar SRC plantations under Mediterranean conditions [252,262].

Other management tools derived from models, such as maps of possible zones of production $[47,273,274]$, estimations derived from the use of new technologies $[270,275,276]$, and tools such as reference diagrams, which are particularly useful for both planning and managing this type of crop [277], have also been developed in recent years.

\subsection{Biomass Characterization}

The use of lignocellulosic biomass is not limited to the production of bioenergy. In recent years, a wide range of bioproduct-related options have sprung up, such as biopolymers, bioplastics, and sugar fermentation bioproducts, among others [278-280].

Poplar lignocellulosic biomass is mainly composed of cellulose (42-49\%), hemicellulose (16-23\%), and lignin $(21-29 \%)$ [22,233,279]. Its biomass can supply raw material for processes of thermochemical or microbiological conversion. Worthy of note among the former is combustion, either for domestic or agro-industrial applications, but also for industrial cogeneration or co-firing [281,282]. Thermochemical processes also include gasification in downdraft gasifiers or fluidized beds [283], slow pyrolysis for the production of biochar [284], and fast pyrolysis to produce biocrude oil [285]. With regards to the biochemical processes, the conversion to second-generation bioethanol is the most studied 
process [286-288], although lignocellulosic biomass can also be a source of biobutanol $[289,290]$ and other second-generation biofuels [22,279,291].

In Spain, several studies have addressed the characterization of poplar biomass for thermal use $[148,231,292,293]$ as biofuels [294-297] or for new bioproducts [298,299]. The lower heating value, which is used to calculated the available energy, is usually around $18-20 \mathrm{MJ} \mathrm{kg}^{-1}$ in poplar wood on a dry basis [300,301]. Values found in Spain are within a range very similar to those obtained for other European countries, although the characteristics vary, depending on the genotype and the age, ranging from 17.61 to $18.74 \mathrm{MJ} \mathrm{kg}^{-1}[137,302,303]$.

The humidity content can be as high as $48-50 \%$ at winter harvesting [231,303], but poplar biomass has shown a good ability for air drying [303]. The specific density is known to be low [304-307], thus deriving in low bulk density chips (150-260 $\mathrm{kg} \mathrm{m}^{-3}$, [231]), corresponding to low energy densities. Densification to produce pellets is therefore an option.

The ash content, which is negatively related to the energy value and associated with the risk of boiler corrosion [308], varies broadly (1-4\%), depending on genotype and site conditions $[117,137,231,303]$. The fouling and slagging risk derived from ash compositions are known to be very low [308-310]. The presence of nitrogen $(\mathrm{N})$, which is related to NOx emissions, is low $(0.5 \%)$ in poplar biomass $[148,231,311]$, along with chlorine $(\mathrm{Cl},<0.02 \%)$ and sulfur $(\mathrm{S},<0.04 \%)$, which are corrosive $[145,312,313]$.

The combination of a high volatile matter content (much higher than $80 \%,[137,303,314]$ ), softness of the wood (which is easy to grind), and a low lignin content makes poplar from SRC a promissory feedstock for gasifiers. It can also be used for the generation of bioethanol [287,315].

Lignocellulosic biomass has many other uses in the context of the bioeconomy. For example, cellulose is used in the manufacturing of cosmetics, textiles, and pharmaceutical products, among others [291,297]. Lignin can potentially be used as a raw material in the manufacturing of products with high value added, such as vanillin, biopolymers in petrochemistry, and biopesticides, as well as a material for soil enrichment [291]. Hyd-Poplar lignin could be used for the production of flame-retardant materials [299]. For example, Martín-Sampedro et al. [234] and Ibarra et al. [233] identified the genotype 'Viriato' as being very promising for use in the production of biofuels, as well as in other value-added products, all of which point to the suitability of poplar raw material for different uses in the context of the bioeconomy.

\subsection{Sustainability and Ecosystem Services}

The importance of the sustainability of crops destined for biomass production has been highlighted when evaluating their future development. During the last decade, the European Commission has carried out different analyses and much effort has been dedicated to defining criteria and indicators of sustainability (EC, Directive 2009/28/EC, 2009; EU Parliament Resolution 2013; Directive 2018/2001/EC; European Parliament 2017). The World Bioenergy Association [40] has drawn up a document which includes 24 voluntary sustainability indicators related to bioenergy in general. This document is particularly important since it represents the only multilateral initiative with a broad consensus among the different governments and international organizations, providing a framework for future policy development. Spain has been part of this association since 2008 through the Institute for Diversification and Energy Saving (IDAE). Despite this consensus, there are many different approaches to tackling these studies, sometimes because of geographical differences, which often leads to approaches that are not always homogeneous. Examples of these initiatives include the analyses conducted by Dallemand et al. [316] and Dimitriou and Rutz [317], among many others.

In any case, the criteria and indicators must be based on scientific evidence and contain specifications for each production model or source of biomass, as these vary considerably. In this regard, there are numerous aspects which need to be addressed, such as life cycle and water cycle analyses, soil quality, and erosion control, among many others [318]. The environmental impacts of establishment, harvesting, and transportation have been considered negligible when the crop is grown 
on marginal land in central Europe [319]. Sustainability is also necessary from economic and energetic perspectives. With regards to the latter, the adaptation would appear to be favorable. However, this may not be the case with regard to the economic viability given the current prices of biomass and absence of subsidies [320,321]. In Spain, several life cycle analyses including not only environmental, but also energetic and even economic, issues [128,237,322-325] have been carried out in order to identify the most relevant factors for these crops under Mediterranean conditions. These analyses concluded that fertilization, transport, and irrigation are some of the most influential factors $[237,288,326,327]$. The price of biomass, the price of land rent, harvesting, and irrigation have been identified as the most influential factors from an economic perspective in Mediterranean environments [214,237,328]. The economic viability of poplar SRC under irrigated Mediterranean conditions can be achieved either by ensuring optimum productivity or through an increase in the market prices, associated with more diversified energy use or bioproducts, along with the quantification of ecosystem services, which currently do not have a market price [214]. In addition to this, improvements in clonal selection and irrigation technology, as well as the employment of other irrigation methods, such as making use of reused water, will be essential if these plantations are to be profitable.

A review by Li et al. [329] highlights the necessity to identify sustainable sources of bioenergy. With this purpose in mind, the authors identified poplar crops among the five lignocellulosic crops with the greatest future potential. They compiled and described biomass yield information across a whole range of locations and countries, and concluded that these crops, which only account for 3\% of the bioenergy in Europe [330], can be grown under a wide range of climatic conditions, thus allowing direct competition with food crops to be avoided. Moreover, it has been determined that woody crops cultivated in short rotation and herbaceous lignocellulosic crops emit between $40 \%$ and $99 \%$ less $\mathrm{NO}_{2}$ than traditional crops and consequently have lower fertilization requirements and a greater $\mathrm{N}$ use efficiency. They also sequester carbon in the biomass that remains in the soil $\left(0.44 \mathrm{Mg} \mathrm{C} \mathrm{ha}^{-1}\right.$ year $\left.^{-1}\right)$ when they are planted on disused agricultural land, although the balance is not positive when they are planted on former pastureland. Other associated ecosystem benefits include an increased biodiversity (phytodiversity and zoological diversity) [32,331], erosion control and soil conservation [332], improvements in the water quality [333], and the role of the crop in phytoremediation [334-336]. In the case of Spain, the potential of the genus in the phytoremediation of soils has been evaluated through examples of the restoration of coal mining areas in northern Spain [131,337,338], or water phytodepuration $[42,187,216,339]$. Research has also focused on nutrient fluxes, evaluating the role of annual leaf litter in soil fertility throughout the rotation [340]; the quantification of accumulated carbon in both above- and below-ground fractions (reaching values of around $6.5 \mathrm{Mg} \mathrm{C} \mathrm{ha-1}$ year $^{-1}$ in the above-ground woody biomass, around $1.0 \mathrm{Mg} \mathrm{C} \mathrm{ha}^{-1}$ year $^{-1}$ in the below-ground biomass [262], and around $2.5 \mathrm{Mg} \mathrm{C} \mathrm{ha}^{-1}$ year $^{-1}$ in the case of the litter [341]), as well as the economic implications of $\mathrm{CO}_{2}$ capture [342]. The impact of these forestry crops on the landscape of the agricultural environments where they are grown has not yet been evaluated in our country. Their effects on soil, biodiversity, and its function of mitigating diffuse pollution are examples of aspects that should be considered.

Among the main concerns in Europe in terms of the sustainability of crops destined for biomass production and therefore SRC plantations, are those linked to land use change. According to some studies, the balance of greenhouse gases and $\mathrm{C}$ in the soil indicates that bioenergy as a whole plays a role in the mitigation of climate change [343]. However, other studies question this affirmation [330], or point to a minimum cultivation period, beyond which the C balance becomes positive [344-347].

The impact on water resources must also be rigorously assessed [332,348]. In the Mediterranean area, the need for irrigation is perceived as one of the main weaknesses of these plantations. Different options aimed at minimizing or achieving more efficient irrigation in Mediterranean environments have been mentioned in Section 3.3.4. The application of wastewater in poplar plantations not only constitutes an attractive method for producing biomass through the regeneration of wastewater, but also implies a reduction or suspension of fertilizer application $[42,187,188]$. Hence, this approach 
not only provides a sustainable way to minimize water use, but also takes advantage of the ability of these plantations as vegetation filters.

Improving the sustainability of SRC crops as contributors to the biomass pool will probably require global decisions that take into account local specifications.

\section{Current Status and Future Prospects in Spain}

The availability of abandoned agricultural land, the lack of economic alternatives in rural areas, and the possibilities for complementarity afforded by SRC crops in terms of supply along with the ecosystem services associated with their establishment on agriculture land, are factors favoring the implementation of SRC forest crops [349-351]. As with other sources of biomass, it is important to analyse the biomass produced from forest crops and assess its sustainability and the best methods for producing it. However, the positive and negative aspects of this type of biomass production, indeed of biomass production in general, must always be taken into account and there will always be a certain degree of controversy surrounding its viability. Biomass from forest crops in short rotation has attracted a lot of interest in Spain, probably due to the regulations regarding electricity production in 2004 (RD 436/2004) and later in 2007 (RD 661/2007). At that time, many large and medium-sized companies considered establishing forest crops in SRC. However, the amendment to this regulation in 2013 removed the incentives at a time when many aspects associated with the establishment of crops in Mediterranean environments had still not been clarified, the ecosystem services had not been assessed, and the economic viability was far from guaranteed. This situation resulted in a declining interest in the sector, which continues today.

Despite the expected gradual increase in the contribution of renewable energy to the final gross energy consumption, in 2017, Spain was still 2.5\% points from its 2020 national target [352]. Of the total contribution of renewable energy, biomass accounts for 13\% [353]. With regards to the amount of biomass destined for bioproducts, as far as we know, there are no statistics for the country given that there are only a residual number of biorefineries producing such bioproducts [280]. Therefore, many aspects must still be resolved before the economic viability of renewable biomass resources can be determined. For example, the price of biomass in Spain is lower than in neighboring countries, so part of the biomass is exported. This would appear to be problematic in terms of sustainability. Moreover, no stable, predictable market for biomass exists in Spain.

In relation to biomass from forest crops in short rotation, many advances have been made in Spain over recent years, which have been described in this article. These advances include the following: (i) Increased knowledge of the genetic material in relation to the environment, with a better understanding of the adaptability of genotypes, including their water-use efficiency; (ii) maximizing production based on densities, genotypes, and management practices; (iii) evaluating the sustainability of plantations from different perspectives (environmental, energetic, and economic); (iv) exploring alternative designs, such as mixed plantations; (v) implementing plantations in marginal areas previously used for mining; (vi) quantifying accumulated carbon; (vii) specific predictive models and management tools for SRC plantations under Mediterranean conditions; (viii) physiological and molecular characterization of attributes, mainly those that are relevant to cultivation in marginal zones; (ix) chemical composition and pyrolytic behavior; and (x) the use of biotechnology to develop new materials, which should lead to increased production in the future. The role of these forest crops in water purification has also recently emerged as a matter of interest.

Despite these advances, we still have a long way to go to make SRC plantations a commercial reality in Spain. It is also known that biomass, including dedicated crops, would generate significant economic returns in rural areas [354]. This may be especially relevant in a country like Spain, where a large part of the country suffers from high levels of depopulation, although some aspects that hinder the short-term economic viability have also been identified, such as those previously mentioned regarding land rental, the need for irrigation, and the technological development of harvesting machinery at reasonable local prices. However, other issues need to be addressed in relation to crop management, 
such as (i) the use of irrigation, which should only be resorted to in areas where it is sustainable, by modernizing the systems, using more water-use efficient plant material or by using recycled water; (ii) the continual updating of genetic material adaptations to the site, testing of new materials, and making use of new technologies (genome editing, linkage maps, etc.), in order to produce material that is resistant to pests and diseases, tolerant to drought or a high salinity, or can adapt more effectively to specific soil and climate conditions; (iii) exploring new plantation designs; although the preliminary results of using mixtures are not particularly encouraging, many different alternatives remain to be explored; and (iv) rationalizing fertilization by evaluating the inputs derived from leaf litter and the exports of nutrients from wood, as well as by using alternative fertilizer inputs, such as those derived from sewage sludge.

Other questions should be explored based on the need to (v) quantify and value ecosystem services in terms of increased biodiversity in the agricultural landscape; carbon accumulation in each of the biomass fractions, both those that are extracted, as well as those that remain in the soil, such as foliar and root biomass; and their additional role in phytoremediation, or (vi) advance predictive modeling for Mediterranean conditions by combining the best features of empirical and process models, the advantages of which have been well-documented. The inclusion of ecophysiological variables enabling the prediction of individual tree-level biometry under different conditions, along with improvements in determining the most important variables, are some of the future objectives of modeling SRC plantations under Mediterranean conditions. In addition, as water restrictions represent a real threat, this factor is also being considered in the development of models that enable the simulation of different climate change scenarios. Regarding (vii) the characterization of biomass, although many studies have already addressed this aspect, a more in-depth knowledge will be required as the final products and their intended uses become more defined. Identifying the requirements of each of the final products will be essential in order to determine the most suitable genotypes for each crop. Given that Spain is a diverse country, it is also necessary (viii) to analyse where and how this biomass is produced at a national scale.

Improving the critical aspects detected in environmental, energetic, and economic analyses is essential for achieving profitable and sustainable plantations under Mediterranean conditions. Biomass produced ad hoc through plantations under SRC systems may be of interest in many areas of the Mediterranean, providing a further option which could contribute to the development of a circular bioeconomy while also generating important environmental services.

Author Contributions: Conceptualization, N.O. and H.S.; funding acquisition, H.S., R.R.-S., and I.C.; investigation, N.O., H.S., C.P.-C., R.R.-S., and I.C.; methodology, H.S. and N.O.; project administration, H.S., R.R.-S., and I.C.; supervision, H.S., I.C., C.P.-C., and R.R.-S.; writing-original draft, N.O. and H.S.; writing-review and editing, N.O., C.P.-C., I.C., R.R.-S., and H.S. All authors have read and agreed to the published version of the manuscript.

Funding: This research was funded by the Ministry of Science and Innovation (Spain) through the framework of the INIA projects RTA2017-00015-CO2, co-financed with funds from FEDER.

Acknowledgments: The authors wish to thank Adam Collins for his English language review of the manuscript.

Conflicts of Interest: The authors declare no conflict of interest.

\section{References}

1. European Commission. The European Green Deal; European Commission: Brussels, Belgium, 2019; p. 24.

2. Hetemäki, L.; Hanewinkel, M.; Muys, B.; Ollikainen, M.; Palahí, M.; Trasobares, A. Leading the Way to a European Circular Bioeconomy Strategy; Science to Policy 5; European Forest Institute: Joensuu, Finland, 2017; Volume 5.

3. European Commission. Innovating for Sustainable Growth: A Bioeconomy for Europe; European Commision: Brussels, Belgium, 2012.

4. European Commission. Review of the 2012 European Bioeconomy Strategy; European Commission: Brussels, Belgium, 2018. 
5. MINECO. Estrategia Española de Bioeconomía: Horizonte 2030; Ministerio de Economía y Competitividad, Secretaría de Estado de Investigación, Desarrollo e Innovación: Madrid, Spain, 2015; p. 46.

6. MITECO. Law on Climate Change and Energy Transition; Ministerio para la Transición Ecológica y el Reto Demográfico: Madrid, Spain, 2020.

7. Bioplat. Agenda Estratégica de Investigación e Innovación. Biomasa y Bioeconomía; Ministerio de Ciencia e Innovación, Gobierno de España: Madrid, Spain, 2020.

8. Sancho, A. Día de la Bioenergía en España: 28 días de Independencia Energética; Bioenergy International: Stockholm, Sweden, 2018.

9. Gardiner, E.S.; Ghezehei, S.B.; Headlee, W.L.; Richardson, J.; Soolanayakanahally, R.Y.; Stanton, B.J.; Zalesny, R.S., Jr. The 2018 Woody Crops International Conference, Rhinelander, Wisconsin, USA, 22-27 July 2018. Forests 2018, 9, 693. [CrossRef]

10. Broeckx, L.; Verlinden, M.; Vangronsveld, J.; Ceulemans, R. Importance of crown architecture for leaf area index of different Populus genotypes in a high-density plantation. Tree Physiol. 2012, 32, 1214-1226. [CrossRef] [PubMed]

11. Huber, J.A.; May, K.; Hülsbergen, K.-J. Allometric tree biomass models of various species grown in short-rotation agroforestry systems. Eur. J. For. Res. 2017, 136, 75-89. [CrossRef]

12. Weih, M. Intensive short rotation forestry in boreal climates: Present and future perspectives. Can. J. For. Res. Rev. Can. Rech. For. 2004, 34, 1369-1378. [CrossRef]

13. Aylott, M.J.; Casella, E.; Tubby, I.; Street, N.R.; Smith, P.; Taylor, G. Yield and spatial supply of bioenergy poplar and willow short-rotation coppice in the UK. New Phytol. 2008, 178, 358-370. [CrossRef]

14. Berhongaray, G.; Verlinden, M.S.; Broeckx, L.S.; Janssens, I.A.; Ceulemans, R. Soil carbon and belowground carbon balance of a short-rotation coppice: Assessments from three different approaches. Glob. Chang. Biol. Bioenergy 2016, 9, 299-313. [CrossRef]

15. European Commission. State of Play on the Sustainability of Solid and Gaseous Biomass Used for Electricity, Heating and Cooling in the EU (SWD 259 Final); European Commission: Brussels, Belgium, 2014.

16. Dimitriou, I.; Berndes, G.; Englund, O.; Murphy, F. Lignocellulosic Crops in Agricultural Landscapes: Production Systems for Biomass and Other Environmental Benefits-Examples, Incentives, and Barriers; IEA Bioenergy: Paris, France, 2018.

17. Dickmann, D.I. An overview of the genus Populus. In Poplar Culture in North America; Dickmann, D.I., Isebrands, J.G., Eckenwalder, J.E., Richardson, J., Eds.; NRC Research Press: Ottawa, ON, Canada, 2001; pp. 1-42.

18. Al Afas, N.; Marron, N.; Van Dongen, S.; Laureysens, I.; Ceulemans, R. Dynamics of biomass production in a poplar coppice culture over three rotations (11 years). For. Ecol. Manag. 2008, 255, 1883-1891. [CrossRef]

19. Laureysens, I.; Pellis, A.; Willems, J.; Ceulemans, R. Growth and production of a short rotation coppice culture of poplar. III. Second rotation results. Biomass Bioenergy 2005, 29, 10-21. [CrossRef]

20. Ceulemans, R.; McDonald, A.; Pereira, J. A comparison among eucalypt, poplar and willow characteristics with particular reference to a coppice, growth-modelling approach. Biomass Bioenergy 1996, 11, $215-231$. [CrossRef]

21. Patel, B.; Gami, B. Biomass characterization and its use as solid fuel for combustion. Iran. J. Energy Environ. 2012, 3, 123-128. [CrossRef]

22. Zhang, X.; Tu, M.; Paice, M. Routes to Potential Bioproducts from Lignocellulosic Biomass Lignin and Hemicelluloses. Bioenergy Res. 2011, 4, 246-257. [CrossRef]

23. Surendran Nair, S.; Kang, S.; Zhang, X.; Miguez, F.E.; Izaurralde, R.C.; Post, W.M.; Dietze, M.C.; Lynd, L.R.; Wullschleger, S.D. Bioenergy crop models: Descriptions, data requirements, and future challenges. Glob. Chang. Biol. Bioenergy 2012, 4, 620-633. [CrossRef]

24. Rodrigues, A.; Loureiro, L.; Nunes, L.J.R. Torrefaction of woody biomasses from poplar SRC and Portuguese roundwood: Properties of torrefied products. Biomass Bioenergy 2018, 108, 55-65. [CrossRef]

25. Schultz, R.; Isenhart, T.; Simpkins, W.; Colletti, J. Riparian forest buffers in agroecosystems-lessons learned from the Bear Creek Watershed, central Iowa, USA. Agrofor. Syst. 2004, 61, 35-50. [CrossRef]

26. Tufekcioglu, A.; Raich, J.W.; Isenhart, T.M.; Schultz, R.C. Fine root dynamics, coarse root biomass, root distribution, and soil respiration in a multispecies riparian buffer in Central Iowa, USA. Agrofor. Syst. 1998, 44, 163-174. [CrossRef] 
27. Zalesny, R.S., Jr.; Stanturf, J.A.; Gardiner, E.S.; Perdue, J.H.; Young, T.M.; Coyle, D.R.; Headlee, W.L.; Banuelos, G.S.; Hass, A. Ecosystem services of woody crop production systems. Bioenergy Res. 2016, 9, 465-491. [CrossRef]

28. Coleman, M.D.; Friend, A.L.; Kern, C.C. Carbon allocation and nitrogen acquisition in a developing Populus deltoides plantation. Tree Physiol. 2004, 24, 1347-1357. [CrossRef]

29. Grigal, D.F.; Berguson, W.E. Soil carbon changes associated with short-rotation systems. Biomass Bioenergy 1998, 14, 371-377. [CrossRef]

30. Baum, S.; Bolte, A.; Weih, M. Short rotation coppice (SRC) plantations provide additional habitats for vascular plant species in agricultural moisac landscapes. Bioenerg. Res. 2012, 5, 573-583. [CrossRef]

31. Christian, D.P.; Niemi, G.J.; Hanowski, J.M.; Collins, P. Perspectives on biomass energy tree plantations and changes in habitat for biological organisms. Biomass Bioenergy 1994, 6, 31-39. [CrossRef]

32. Vanbeveren, S.P.P.; Ceulemans, R. Biodiversity in short-rotation coppice. Renew. Sustain. Energy Rev. 2019, 111, 34-43. [CrossRef]

33. Ferrarini, A.; Serra, P.; Almagro, M.; Trevisan, M.; Amaducci, S. Multiple ecosystem services provision and biomass logistics management in bioenergy buffers: A state-of-the-art review. Renew. Sustain. Energy Rev. 2017, 73, 277-290. [CrossRef]

34. Rockwood, D.; Naidu, C.; Carter, D.; Rahmani, M.; Spriggs, T.; Lin, C.; Alker, G.; Isebrands, J.; Segrest, S. Short-rotation woody crops and phytoremediation: Opportunities for agroforestry? In New Vistas in Agroforestry. A Compendium for the 1st World Congress of Agroforestry; Kluwer Academic Publisher: Dordrecht, The Netherlands; Boston, MA, USA; New York, NY, USA, 2004; pp. 51-63.

35. Dipesh, K.; Will, R.E.; Hennessey, T.C.; Penn, C.J. Evaluating performance of short-rotation woody crops for bioremediation purposes. New For. 2015, 46, 267-281. [CrossRef]

36. Pilipović, A.; Zalesny, R.S., Jr.; Orlović, S.; Drekić, M.; Pekeč, S.; Katanić, M.; Poljaković-Pajnik, L. Growth and physiological responses of three poplar clones grown on soils artificially contaminated with heavy metals, diesel fuel, and herbicides. Int. J. Phytoremediat. 2020, 22, 436-450. [CrossRef] [PubMed]

37. Zalesny, R.S., Jr.; Stanturf, J.A.; Gardiner, E.S.; Bañuelos, G.S.; Hallett, R.A.; Hass, A.; Stange, C.M.; Perdue, J.H.; Young, T.M.; Coyle, D.R. Environmental technologies of woody crop production systems. Bioenergy Res. 2016, 9, 492-506. [CrossRef]

38. Evangelou, M.W.; Conesa, H.M.; Robinson, B.H.; Schulin, R. Biomass production on trace elementcontaminated land: A review. Biomass Bioenergy 2012, 29, 823-839. [CrossRef]

39. IRENA. Global Renewables Outlook: Energy Transformation 2050; International Renewable Energy Agency: Abu Dhabi, UAE, 2020; p. 212.

40. GBEP. The Global Bioenergy Partnership Sustainability Indicators for Bioenergy; Climate, Energy and Tenure Division, Food and Agriculture Organization of the United Nations (FAO): Rome, Italy, 2011.

41. Marron, N. Agronomic and environmental effects of land application of residues in short-rotation tree plantations: A literature review. Biomass Bioenergy 2015, 81, 378-400. [CrossRef]

42. de Miguel, A.; Meffe, R.; Leal, M.; González-Naranjo, V.; Martínez-Hernández, V.; Lillo, J.; Martín, I.; Salas, J.J.; Bustamante, I.d. Treating municipal wastewater through a vegetation filter with a short-rotation poplar species. Ecol. Eng. 2014, 73, 560-568. [CrossRef]

43. Zalesny, R.S., Jr.; Stanturf, J.A.; Evett, S.R.; Kandil, N.F.; Soriano, C. Opportunities for woody crop production using treated wastewater in Egypt. I. Afforestation strategies. Int. J. Phytoremediat. 2011, 13, 102-121. [CrossRef]

44. San Miguel, A.; Montoya, J. Resultados de los primeros 5 años de produccion de talleres de chopo en rotacion corta (2-5 anõs). In Anales del Instituto Nacional de Investigaciones Agrarias. Serie Forestal; Instituto Nacional de Investigaciones Agrarias: Madrid, Spain, 1984; pp. 73-91.

45. Marcos, F. Cultivos energéticos forestales. In Proceedings of the V Conferencia sobre Planificación, Ahorro y Alternativas Energéticas, Zaragoza, Spain, 1985.

46. Ciria, M.P.; Mazon, P.; Carrasco, J. Productivity of poplar grown on short rotation in Spain: Influence of the rotation age and plant density. In Proceedings of the 2nd biomass conference of the Americas: Energy, Environment, Agriculture, and Industry, Portland, OR, USA, 21-24 August 1995; pp. 235-243.

47. Pérez-Cruzado, C.; Sanchez-Ron, D.; Rodríguez-Soalleiro, R.; Hernández, M.; Sánchez-Martín, M.; Cañellas, I.; Sixto, H. Biomass production assessment from Populus spp. short-rotation irrigated crops in Spain. Glob. Chage Biol. Bioenergy 2013, 6, 312-326. [CrossRef] 
48. Huber, J.A.; May, K.; Siegl, T.; Schmid, H.; Gerl, G.; Hülsbergen, K.J. Yield potential of tree species in organic and conventional short-rotation agroforestry systems in Southern Germany. Bioenergy Res. 2016, 9, 955-968. [CrossRef]

49. Havlíčková, K.; Weger, J.; Zánová, I. Short rotation coppice for energy purposes-Economy conditions and landscape functions in the Czech Republic. In Proceedings of ISES World Congress 2007; Springer: Berlin/Heidelberg, Germany, 2008; pp. 2482-2487.

50. Stankova, T.; Gyuleva, V.; Tsvetkov, I.; Popov, E.; Velinova, K.; Velizarova, E.; Dimitrov, D.N.; Hristova, H.; Kalmukov, K.; Dimitrova, P. Aboveground dendromass allometry of hybrid black poplars for energy crops. Ann. For. Res. 2016, 59, 61-74. [CrossRef]

51. Pilipović, A.; Orlović, S.; Kovačević, B.; Galović, V.; Stojnić, S. Selection and Breeding of Fast Growing Trees for Multiple Purposes in Serbia. In Forests of Southeast Europe Under a Changing Climate; Springer: Berlin/Heidelberg, Germany, 2019; pp. 239-249.

52. Niemczyk, M.; Kaliszewski, A.; Jewiarz, M.; Wróbel, M.; Mudryk, K. Productivity and biomass characteristics of selected poplar (Populus spp.) cultivars under the climatic conditions of northern Poland. Biomass Bioenergy 2018, 111, 46-51. [CrossRef]

53. Benbrahim, M.; Gavaland, A.; Gauvin, J. Growth and yield of mixed polyclonal stands of Populus in short-rotation coppice. Scand. J. For. Res. 2000, 15, 605-610. [CrossRef]

54. Nardin, F.; Alasia, F. Use of selected fast growth poplar trees for a woody biomass production die along Po valley. In Proceedings of the 2nd World Conference on Biomass for Energy, Industry and Climate Protection, Rome, Italy, 10-14 May 2004; pp. 247-249.

55. Sixto, H.; Gil, P.; Ciria, P.; Camps, F.; Sánchez, M.; Cañellas, I.; Voltas, J. Performance of hybrid poplar clones in short rotation coppice in Mediterranean environments: Analysis of genotypic stability. Bioenegy 2013, 6, 661-671. [CrossRef]

56. Nordborg, M.; Berndes, G.; Dimitriou, I.; Henriksson, A.; Mola-Yudego, B.; Rosenqvist, H. Energy analysis of poplar production for bioenergy in Sweden. Biomass Bioenergy 2018, 112, 110-120. [CrossRef]

57. Tullus, A.; Rosenvald, K.; Lutter, R.; Kaasik, A.; Kupper, P.; Sellin, A. Coppicing improves the growth response of short-rotation hybrid aspen to elevated atmospheric humidity. Forest Ecol. Manag. 2020, 459, 117825. [CrossRef]

58. Clifton-Brown, J.; Harfouche, A.; Casler, M.D.; Dylan Jones, H.; Macalpine, W.J.; Murphy-Bokern, D.; Smart, L.B.; Adler, A.; Ashman, C.; Awty-Carroll, D. Breeding progress and preparedness for mass-scale deployment of perennial lignocellulosic biomass crops switchgrass, miscanthus, willow and poplar. Glob. Chang. Biol. Bioenergy 2019, 11, 118-151. [CrossRef]

59. Scholz, V.; Ellerbrock, R. The growth productivity, and environmental impact of the cultivation of energy crops on sandy soil in Germany. Biomass Bioenergy 2002, 23, 81-92. [CrossRef]

60. Zhang, S.Y.; Yu, Q.; Chauret, G.; Koubaa, A. Selection for both growth and wood properties in hybrid poplar clones. For. Sci. 2003, 49, 901-908. [CrossRef]

61. Tuskan, G.A.; Difazio, S.; Jansson, S.; Bohlmann, J.; Grigoriev, I.; Hellsten, U.; Putnam, N.; Ralph, S.; Rombauts, S.; Salamov, A. The genome of black cottonwood, Populus trichocarpa (Torr. \& Gray). Science 2006, 313, 1596-1604. [CrossRef] [PubMed]

62. FAO. Working Party on Sustainable Livelihoods, Land-Use, Products and Bioenergy. Available online: http://www.fao.org/forestry/ipc/69631/en/ (accessed on 15 September 2020).

63. Glas, D.; Schimer, R. Prüfung von Pappelsorten aus Anderen EU-Staaten für Kurzumtriebsplantagen (EU-POP); Bundesministerium für Ernährung und Landwirtschaft: Teisendorf, Germany, 2018.

64. MAPA. Estadísticas Comisión Nacional del Chopo. Available online: https://www.mapa.gob.es/es/desarrollorural/temas/politica-forestal/comision-nacional-del-chopo/estadisticas.aspx (accessed on 20 September 2020).

65. ProPopulus. La Escasez de Chopo Amenaza a la Industria Española de Contrachapado; ProPopulus; Nature, Society, Future; The European Poplar Initiative: Spain, 2019; Available online: http://propopulus.eu/es/la-escasez-dechopo-amenaza-a-la-industria-espanola-de-contrachapado/ (accessed on 10 December 2020).

66. Rueda, J.; García Caballero, J.L.; Cuevas, Y.; García-Jiménez, C.; Villar, C. Cultivo de Chopos en Castilla y León; Consejería de Fomento y Medio Ambiente, Junta de Castilla y León: Valladolid, Spain, 2019.

67. ESYRCE. Encuesta Sobre Superficies y Rendimientos de Cultivos. Informe Sobre Regadíos en España; Subsecretaría de Agricultura, Pesca y Alimentación y Subdirección General de Análisis, Coordinación y Estadística: Madrid, Spain, 2019. 
68. Sixto, H.; Hernández, M.; Barrio, M.; Carrasco, J.; Cañellas, I. Plantaciones del género Populus para la producción de biomasa con fines energéticos: Revisión. For. Syst. 2007, 16, 277-294.

69. Corcuera, L.; Maestro, C.; Notivol, E. La ecofisiología como herramienta para la selección de clones más adaptados y productivos en el marco de una selvicultura clonal con chopos. Invest. Agrar. Sist. Recur. For. 2005, 14, 394-407. [CrossRef]

70. Pérez-Cruzado, C.; Merino, A.; Rodríguez-Soalleiro, R. A management tool for estimating bioenergy production and carbon sequestration in Eucalyptus globulus and Eucalyptus nitens grown as short rotation woody crops in north-west Spain. Biomass Bioenerg 2011, 35, 2839-2851. [CrossRef]

71. Pita, P.; Soria, F.; Canas, I.; Toval, G.; Pardos, J. Carbon isotope discrimination and its relationship to drought resistance under field conditions in genotypes of Eucalyptus globulus Labill. Forest Ecol. Manag. 2001, 141, 211-221. [CrossRef]

72. Valdés, A.E.; Irar, S.; Majada, J.P.; Rodríguez, A.; Fernández, B.; Pagès, M. Drought tolerance acquisition in Eucalyptus globulus (Labill.): A research on plant morphology, physiology and proteomics. J. Proteom. 2013, 79, 263-276. [CrossRef] [PubMed]

73. Perez, I.; Perez, J.; Carrasco, J.; Ciria, P. Siberian elm responses to different culture conditions under short rotation forestry in Mediterranean areas. Turk. J. Agric. For. 2014, 38, 652-662. [CrossRef]

74. Manzone, M.; Bergante, S.; Facciotto, G. Energy and economic sustainability of woodchip production by black locust (Robinia pseudoacacia L.) plantations in Italy. Fuel 2015, 140, 555-560. [CrossRef]

75. Böhm, C.; Quinkenstein, A.; Freese, D. Yield prediction of young black locust (Robinia pseudoacacia L.) plantations for woody biomass production using allometric relations. Ann. For. Res. 2011, 54, $215-227$.

76. Oliveira, N.; del Río, M.; Forrester, D.I.; Rodríguez-Soalleiro, R.; Pérez-Cruzado, C.; Cañellas, I.; Sixto, H. Mixed short rotation plantations of Populus alba and Robinia pseudoacacia for biomass yield. For. Ecol. Manag. 2018, 410, 48-55. [CrossRef]

77. Sixto, H.; Cañellas, I.; van Arendonk, J.; Ciria, P.; Camps, F.; Sánchez, M.; Sánchez-González, M. Growth potential of different species and genotypes for biomass production in short rotation in Mediterranean environments. For. Ecol. Manag. 2015, 354, 291-299. [CrossRef]

78. Rosso, L.; Facciotto, G.; Bergante, S.; Vietto, L.; Nervo, G. Selection and testing of Populus alba and Salix spp. as bioenergy feedstock: Preliminary results. Appl. Energy 2013, 102, 87-92. [CrossRef]

79. Karp, A.; Shield, I. Bioenergy from plants and the sustainable yield challenge. New Phytol. 2008, 179, 15-32. [CrossRef] [PubMed]

80. Oliveira, N.; Ciria, P.; Camps, F.; Sánchez-González, M.; Sánchez, M.; Cañellas, I.; Sixto, H. Assessing genotypes in second rotation for lignocellulosic biomass production. In Proceedings of the 23rd European Biomass Conference and Exhibition (EUBCE), Viena, Austria, 1-4 June 2015.

81. Sixto, H.; Calvo, R.; Sánchez, M.M.; Arrieta, J.A.; Otero, J.M.; Salvia, J.; Cañellas, I. Fine scale site variation correlated to growth in a Salicaceae plantations (Salix and Populus) during the first vegetative period. In Proceedings of the 24th International Poplar Commission. Improving lives with poplars and willows, Dehradun, India, 30 October-2 November 2012.

82. Zuazo, V.H.D.; Bocanegra, J.A.J.; Torres, F.P.; Pleguezuelo, C.R.R.; Martínez, J.R.F. Biomass yield potential of Paulownia trees in a semi-arid Mediterranean environment (Spain). Int. J. Renew. Energy Res. 2013, 3, 789-793.

83. García-Morote, F.A.; López-Serrano, F.R.; Martínez-García, E.; Andrés-Abellán, M.; Dadi, T.; Candel, D.; Rubio, E.; Lucas-Borja, M.E. Stem biomass production of Paulownia elongata $\times$ P. fortunei under low irrigation in a semi-arid environment. Forests 2014, 5, 2505-2520. [CrossRef]

84. Hernández Garasa, M.J.; Sixto, H.; Ciria, P.; Carrasco, J.; Cañellas, I. Paulownia plantations for bioenergy in Spain. In Proceedings of the 16th European Biomass confernce and Exhibition, Valencia, Spain, 2-6 May 2008.

85. Bradshaw, A.D. Evolutionary significance of phenotypic plasticity in plants. Adv. Genet. 1965, 13, 115-155.

86. Zalesny, R.S., Jr.; Hall, R.B.; Zalesny, J.A.; McMahon, B.G.; Berguson, W.E.; Stanosz, G.R. Biomass and genotype $\times$ environment interactions of Populus energy crops in the Midwestern United States. Bioenergy Res. 2009, 2, 106-122. [CrossRef]

87. Mohn, C.; Randall, W. Interaction of cottonwood clones with site and planting year. Can. J. For. Res. 1973, 3, 329-332. [CrossRef]

88. Orlovic, S.; Guzina, V.; Krstic, B.; Merkulov, L. Genetic variability in anatomical, physiological and growth characteristics of hybrid poplar (Populus $\times$ euramericana Dode (Guinier)) and eastern cottonwood (Populus deltoides Bartr.) clones. Silvae Genet. 1998, 47, 183-189. 
89. Becker, H.; Leon, J. Stability analysis in plant breeding. Plant Breed. 1988, 101, 1-23. [CrossRef]

90. Bergante, S.; Facciotto, G.; Minotta, G. Identification of the main site factors and management intensity affecting the establishment of Short-Rotation-Coppices (SRC) in Northern Italy through stepwise regression analysis. Cent. Eur. J. Biol. 2010, 5, 522-530. [CrossRef]

91. Li, Y.; Suontama, M.; Burdon, R.D.; Dungey, H.S. Genotype by environment interactions in forest tree breeding: Review of methodology and perspectives on research and application. Tree Genet. Genomes 2017, 13, 60. [CrossRef]

92. Sabatti, M.; Fabbrini, F.; Harfouche, A.; Beritognolo, I.; Mareschi, L.; Carlini, M.; Paris, P.; Scarascia-Mugnozza, G. Evaluation of biomass production potential and heating value of hybrid poplar genotypes in a short-rotation culture in Italy. Ind. Crop. Prod. 2014, 61, 62-73. [CrossRef]

93. Sixto, H.; Gil, P.M.; Ciria, P.; Camps, F.; Cañellas, I.; Voltas, J. Interpreting genotype-by-environment interaction for biomass production in hybrid poplars under short-rotation coppice in Mediterranean environments. Glob. Chang. Biol. Bioenergy 2016, 8, 1124-1135. [CrossRef]

94. Hernández, P.M.G.; Voltas, J.; González, M.S.; Martín, M.M.S.; Camps, F.; Ciria, M.P.; Cañellas, I.; Sixto, H. Análisis de estabilidad en genotipos híbridos de "Populus" L. para producción de biomasa. Cuad. Soc. Esp. Cienc. For. 2012, 105-110.

95. Sixto, H.; Salvia, J.; Barrio, M.; Ciria, M.P.; Canellas, I. Genetic variation and genotype-environment interactions in short rotation Populus plantations in southern Europe. New For. 2011, 42, 163-177. [CrossRef]

96. Stanton, B. Poplar hybridisation and clonal evaluation: James River, Lower Columbia River Fibber Farm, Westport Research Station. In Proceedings of the International Poplar Symposium, Seattle, WA, USA, 20-25 August 1995; pp. 114-115.

97. Fladung, M.; Polak, O. Ac/Ds-transposon activation tagging in poplar: A powerful tool for gene discovery. BMC Genom. 2012, 13, 61. [CrossRef]

98. Marchadier, H.; Sigaud, P. Los álamos en la investigación biotecnológica. Unasylva 2005, 56, 38-39.

99. Fan, D.; Liu, T.; Li, C.; Jiao, B.; Li, S.; Hou, Y.; Luo, K. Efficient CRISPR/Cas9-mediated targeted mutagenesis in Populus in the first generation. Sci. Rep. 2015, 5, 12217. [CrossRef]

100. Zhou, X.; Jacobs, T.B.; Xue, L.J.; Harding, S.A.; Tsai, C.J. Exploiting SNPs for biallelic CRISPR mutations in the outcrossing woody perennial Populus reveals 4-coumarate: CoA ligase specificity and redundancy. New Phytol. 2015, 208, 298-301. [CrossRef]

101. Liu, T.; Fan, D.; Ran, L.; Jiang, Y.; Liu, R.; Luo, K. Highly efficient CRISPR/Cas9-mediated targeted mutagenesis of multiple genes in Populus. Yi Chuan 2015, 37, 1044-1052. [CrossRef] [PubMed]

102. Pilate, G.; Allona, I.; Boerjan, W.; Déjardin, A.; Fladung, M.; Gallardo, F.; Häggman, H.; Jansson, S.; Van Acker, R.; Halpin, C. Lessons from 25 years of GM tree field trials in Europe and prospects for the future. In Biosafety of Forest Transgenic Trees; Springer: Berlin/Heidelberg, Germany, 2016; pp. 67-100.

103. Porth, I.; Klapšte, J.; Skyba, O.; Hannemann, J.; McKown, A.D.; Guy, R.D.; DiFazio, S.P.; Muchero, W.; Ranjan, P.; Tuskan, G.A. Genome-wide association mapping for wood characteristics in Populus identifies an array of candidate single nucleotide polymorphisms. New Phytologist. 2013, 200, 710-726. [CrossRef] [PubMed]

104. Ludovisi, R.; Tauro, F.; Salvati, R.; Khoury, S.; Mugnozza Scarascia, G.; Harfouche, A. UAV-based thermal imaging for high-throughput field phenotyping of black poplar response to drought. Front. Plant Sci. 2017, 8, 1681. [CrossRef] [PubMed]

105. Gömöry, D.; Hrivnák, M.; Krajmerová, D.; Longauer, R. Epigenetic memory effects in forest trees: A victory of "Michurinian biology"? Cent. Eur. For. J. 2017, 63, 173-179. [CrossRef]

106. Le Gac, A.-L.; Lafon-Placette, C.; Chauveau, D.; Segura, V.; Delaunay, A.; Fichot, R.; Marron, N.; Le Jan, I.; Berthelot, A.; Bodineau, G. Winter-dormant shoot apical meristem in poplar trees shows environmental epigenetic memory. J. Exp. Bot. 2018, 69, 4821-4837. [CrossRef]

107. Jing, Z.P.; Gallardo, F.; Pascual, M.B.; Sampalo, R.; Romero, J.; De Navarra, A.T.; Cánovas, F.M. Improved growth in a field trial of transgenic hybrid poplar overexpressing glutamine synthetase. New Phytol. 2004, 164, 137-145. [CrossRef]

108. Conde, D.; Moreno-Cortés, A.; Dervinis, C.; Ramos-Sánchez, J.M.; Kirst, M.; Perales, M.; González-Melendi, P.; Allona, I. Overexpression of DEMETER, a DNA demethylase, promotes early apical bud maturation in poplar. Plant Cell Environ. 2017, 40, 2806-2819. [CrossRef] 
109. Moreno-Cortés, A.; Hernández-Verdeja, T.; Sánchez-Jiménez, P.; González-Melendi, P.; Aragoncillo, C.; Allona, I. CsRAV1 induces sylleptic branching in hybrid poplar. New. Phytol. 2012, 194, 83-90. [CrossRef]

110. Moreno-Cortés, A.; Ramos-Sánchez, J.M.; Hernández-Verdeja, T.; González-Melendi, P.; Alves, A.; Simões, R.; Rodrigues, J.C.; Guijarro, M.; Canellas, I.; Sixto, H. Impact of RAV1-engineering on poplar biomass production: A short-rotation coppice field trial. Biotechnol. Biofuels 2017, 10, 110. [CrossRef]

111. Sixto, H. Populus alba clones and their hybrids growing under water deficit conditions for biomass production in short rotation forestry. In Proceedings of the VI International Poplar Symposium (IUFRO), Vancouver, BC, Canada, 20-28 July 2014.

112. Marcos, F.; Izquierdo, I.; Gracia, R.; Godino, M.; Ruiz, J.; Vilegas, S. Estudio de plantaciones energéticas de chopo (Populus $\times$ euramericana cv. I-214) a turnos muy cortos; Documento interno del Departamento de Ingeniería Forestal ETSI de Montes: Madrid, Spain, 2002.

113. San Miguel, A.; San Miguel, J.; Yagüe, S. Tallares de chopo a turno corto. In Proceedings of the $19^{a}$ Sesión de la Comisión Internacional del Álamo, Zaragoza, Spain, 22-25 September 1992; pp. 143-156.

114. Sixto, H.; Grau, J.M.; García-Baudín, J.M. Assessment of the effect of broad-spectrum pre-emergence herbicides in poplar nurseries. Crop Prot. 2001, 20, 121-126. [CrossRef]

115. Sixto, H.; Barrio, M.; Aranda, I. Evaluación de criterios para la selección de clones de chopo como productores de biomasa. In Proceedings of the XVII Reunión de la Sociedad Española de Fisiología Vegetal y X Congreso Hispano-Luso de Fisiología Vegetal Alcalá de Henares, Madrid, Spain, 18-21 September 2007.

116. Sixto, H.; Grau, J.; Alba, N.; Alia, R. Response to sodium chloride in different species and clones of genus Populus L. Forestry 2005, 78, 93-104. [CrossRef]

117. Álvarez-Álvarez, P.; Pizarro, C.; Barrio-Anta, M.; Cámara-Obregón, A.; Bueno, J.L.M.; Álvarez, A.; Gutiérrez, I.; Burslem, D.F. Evaluation of tree species for biomass energy production in Northwest Spain. Forests 2018, 9, 160. [CrossRef]

118. Castaño Díaz, M. Producción de Biomasa Procedente de Cultivos Energéticos Leñosos en Terrenos Ociosos de Minería en el PRINCIPADO de Asturias. Ph.D. Thesis, Biología de Organismos y Sistemas, Departamento de, University of Oviedo, Oviedo, Spain, 2018.

119. Fiala, M.; Bacenetti, J. Economic, energetic and environmental impact in short rotation coppice harvesting operations. Biomass Bioenergy 2012, 42, 107-113. [CrossRef]

120. Cañellas, I.; Huelin, P.; Hernández, M.; Ciria, P.; Calvo, R.; Gea-Izquierdo, G.; Sixto, H. The effect of density on Short Rotation Populus spp. plantations in the Mediterranean area. Biomass Bioenergy 2012, 46, 645-652. [CrossRef]

121. Hernández Garasa, M.; Montes Pita, F. Evolución del índice de área foliar en plantaciones de chopo con fines energéticos. Comparación entre métodos semidirectos e indirectos de estimación. In Proceedings of the $5^{\circ}$ Congreso Forestal Español, Ávila, Spain, 21-25 September 2009.

122. Sixto, H.; Rueda, J. Evaluación de genotipos para la producción de biomasa con fines energéticos en la Comunidad de Castilla y León. In Proceedings of the $5^{\circ}$ Congresos Forestales, Ávila, Spain, 21-25 September 2009.

123. Klasnja, B.; Orlovic, S.; Drekic, M.; Markovic, M. Energy production from short rotation poplar plantations. In Proceedings of the 7th International Symposium on Interdisciplinary Regional Research, Hungary, Serbia and Montenegro, Hunedoara, Romania, 25-25 September 2003; pp. 161-166.

124. Fiala, M.; Bacenetti, J.; Scaravonati, A.; Bergonzi, A. Short rotation coppice in northern Italy: Comprenhensive Sustainability. In Proceedings of the 18th European Biomass Conference and Exhibition, Lyon, France, 3-7 May 2010; pp. 342-348.

125. DeBell, D.S.; Clendenen, G.W.; Zasadat, J.C. Growing Populus biomass: Comparison of woodgrass versus wider-spaced short-rotation systems. Biomass Bioenergy 1993, 4, 305-313. [CrossRef]

126. Ciria, M.; González, E.; Carrasco, J. The effect of fertilization and planting density on biomass productivity of poplar harvested after three-years rotation. In Proceedings of the 12th European Conference and Technology Exhibition on Biomass for Energy, Industry and Climate Protection, Amsterdam, The Netherlands, 17-21 June 2002; pp. 28-286.

127. Ciria, P. Efecto del Turno de Corta y de la Densidad de Plantación Sobre la Productividad de Diversos Clones de Chopo en Condiciones de Corta Rotación. Ph.D. Thesis, Universidad Politécnica de Madrid, Madrid, Spain, 1999. 
128. Sevigne, E.; Gasol, C.M.; Brun, F.; Rovira, L.; Pagés, J.M.; Camps, F.; Rieradevall, J.; Gabarrell, X. Water and energy consumption of Populus spp. bioenergy systems: A case study in Southern Europe. Renew. Sustain. Energy Rev. 2011, 15, 1133-1140. [CrossRef]

129. Dillen, S.Y.; Djomo, S.N.; Al Afas, N.; Vanbeveren, S.; Ceulemans, R. Biomass yield and energy balance of a short-rotation poplar coppice with multiple clones on degraded land during 16 years. Biomass Bioenergy 2013, 56, 157-165. [CrossRef]

130. Friedrich, E. Anbautechnische Untersuchungen in Forstlichen Schnellwuchsplantagen und Demonstration des Leistungsvermögens Schnellwachsender Baumarten; Landwirtschaftsverlag Münster: Leipzig, Germany, 1999.

131. Menéndez, J.; Loredo Pérez, J.L. Biomass production in surface mines: Renewable energy source for power plants. WSEAS Trans. Environ. Dev. 2018, 14, 205-211.

132. Martín-García, J.; Merino, A.; Diez, J.J. Relating visual crown conditions to nutritional status and site quality in monoclonal poplar plantations (Populus $\times$ euramericana). Eur. J. For. Res. 2012, 131, 1185-1198. [CrossRef]

133. Bungart, R.; Hüttl, R.F. Growth dynamics and biomass accumulation of 8-year-old hybrid poplar clones in a short-rotation plantation on a clayey-sandy mining substrate with respect to plant nutrition and water budget. Eur. J. For. Res. 2004, 123, 105-115. [CrossRef]

134. Truax, B.; Fortier, J.; Gagnon, D.; Lambert, F. Planting density and site effects on stem dimensions, stand productivity, biomass partitioning, carbon stocks and soil nutrient supply in hybrid poplar plantations. Forests 2018, 9, 293. [CrossRef]

135. O Di Nasso, N.N.; Guidi, W.; Ragaglini, G.; Tozzini, C.; Bonari, E. Biomass production and energy balance of a 12 year old short rotation coppice poplar stand under different cutting cycles. Bioenergy 2010, 2, 89-97. [CrossRef]

136. Vanbeveren, S.P.P.; Ceulemans, R. Genotypic differences in biomass production during three rotations of short-rotation coppice. Biomass Bioenergy 2018, 119, 198-205. [CrossRef]

137. Gómez-Martín, J.M.; Castaño-Díaz, M.; Cámara-Obregón, A.; Álvarez-Álvarez, P.; Folgueras-Díaz, M.B.; Diez, M.A. On the chemical composition and pyrolytic behavior of hybrid poplar energy crops from northern Spain. Energy Rep. 2020, 6, 764-769. [CrossRef]

138. Garasa, M.J.H.; Cañellas, I.; Viscasillas, E.; García, A.; Carrasco, J.E.; Sixto, H. Biomass yield in a short rotation clonal poplar trial. In Proceedings of the 18th European Biomass Conference and Exhibitions, Lyon, France, 3-7 May 2010.

139. Oliveira, N.; De la Iglesia, J.P.; Viscasillas, E.; Bachiller, A.; Parras, A.; González, I.; Grau, J.M.; Otero, J.M.; Cañellas, I.; Sixto, H. Adecuación de genotipos para la producción de biomasa en la meseta septentrional. In Proceedings of the II Simposio del Chopo, Valladolid, Spain, 17-19 October 2018.

140. Oliveira, N. Evaluación de la productividad clonal en biomasa, en un cultivo energético experimental de chopo instalado por INIA-CIFOR en Almazán (Soria). Master's Thesis, University of Santiago de Compostela, Lugo, Spain, 2012.

141. Willebrand, E.; Verwijst, T. Population dynamics of willow coppice systems and their implications for management of short-rotation forests. Forest Chron. 1993, 69, 699-704. [CrossRef]

142. Kopp, R.; Abrahamson, L.; White, E.; Volk, T.; Nowak, C.; Fillhart, R. Willow biomass production during ten successive annual harvests. Biomass Bioenergy 2001, 20,1-7. [CrossRef]

143. Tubby, I.; Armstrong, A. Establishment and Management of Short Rotation Coppice. 2002. Available online: https://www.cabdirect.org/cabdirect/abstract/20023172097 (accessed on 15 November 2020).

144. Sixto, H.; Hernández, M.J.; de Miguel, J.; Cañellas, I. Red de Parcelas de Cultivos Leñosos en Alta Densidad y Turno Corto; Instituto Nacional de Investigación y Tecnología Agraria y Alimentaria, Ministerio de Economía y Competitividad: Madrid, Spain, 2013; p. 188.

145. Fernández, M.J.; Barro, R.; Pérez, J.; Losada, J.; Ciria, P. Influence of the agricultural management practices on the yield and quality of poplar biomass (a 9-year study). Biomass Bioenergy 2016, 93, 87-96. [CrossRef]

146. Sixto, H.; Ciria, P.; Rueda, J.; Perez, J.; García Caballero, J.; Mazón, P.; Montoto, J.L.; Cañellas, I. Comparison of two cutting scenarios and clone response in the first rotation period of a poplar crop for biomass energy production. In Proceedings of the 17th European Biomass Conference and Exhibition, Hamburg, Germany, 28 June-3 July 2009.

147. Štochlová, P.; Novotná, K.; Costa, M.; Rodrigues, A. Biomass production of poplar short rotation coppice over five and six rotations and its aptitude as a fuel. Biomass Bioenergy 2019, 122, 183-192. [CrossRef] 
148. Fernández, M.J.; Barro, R.; Pérez, J.; Ciria, P. Production and composition of biomass from short rotation coppice in marginal land: A 9-year study. Biomass Bioenergy 2020, 134, 105478. [CrossRef]

149. Oliveira, N.; Otero, J.M.; Pasalodos, M.; Cañellas, I.; Pereira-Espinel, J.; Rodríguez-Soalleiro, R.; Sixto, H. Analisis de los costes de cultivos forestales de turno corto de chopo en España para produccion de bioenergia. In Proceedings of the XII Congreso de Economía Agraria, Lugo, Spain, 4-6 September 2019.

150. Sixto, H.; Grau, J.M.; Eugenio, M.E.; Ibarra, D.; Hernandez, J.J.; Monedero, E.; Oliveira, N.; Otero, J.M.; Cañellas, I. Evolution of biomass production with poplar hybrids in southern Europe. In Proceedings of the 7th International Poplar Symposium. New Bioeconomies: Exploring the role of Salicaceae, Buenos Aires, Argentina, 28 October-4 November 2018.

151. Dawson, J.O.; Hansen, E.A. Effect of Alnus glutinosa on hybrid populus growth and soil nitrogen concentration in a mixed plantation. In Intensive Plantation Culture: 12 Years Research; Hansen, E.A., Ed.; Gen. Tech. Rep. NC-91; U.S. Department of Agriculture, Forest Service, North Central Forest Experiment Station: St. Paul, MN, USA, 1983; pp. 29-34.

152. Radwan, M.; DeBell, D. Nutrient relations in coppiced black cottonwood and red alder. Plant Soil 1988, 106, 171-177. [CrossRef]

153. Pelleri, F.; Plutino, M.; Manetti, M.C.; Sansone, D.; Bergante, S.; Castro, G.; Moya, J.F.; Chiarabaglio, P.M.; Urban, M.I. Plantaciones policíclicas mixtas: Nogales, chopos y biomasa de rotación corta. In Proceedings of the II Simposio del Chopo, Valladolid, Spain, 17-19 October 2018.

154. Gana, C.; Epron, D.; Gérant, D.; Maillard, P.; Plain, C.; Priault, P.; Marron, N. How does poplar / black locust mixture influence growth and functioning of each species in a short rotation plantation? In Proceedings of the VI International Poplar Symposium, Vancouver, BC, Canada, 20-23 July 2014.

155. Marron, N.; Priault, P.; Gana, C.; Gérant, D.; Epron, D. Prevalence of interspecific competition in a mixed poplar/black locust plantation under adverse climate conditions. Ann. For. Sci. 2018, 75, 23. [CrossRef]

156. Oviedo, P.A.; Gutiérrez, J.A.; Martín, R.T.; Martínez, M.F. Cultivo mixto de especies forestales de turno corto (Robinia pseudoacacia y Populus $\times$ euroamericana clon AF2) con fines energéticos. Cuad. Soc. Esp. Cienc. For. 2016, 17-30. [CrossRef]

157. Oliveira, N.; Del Río, M.; Rodríguez-Soalleiro, R.; Pérez-Cruzado, C.; Cañellas, I.; Sixto, H. Mixed short rotation plantation of Populus alba and Robinia pseudoacacia for biomass yield over the course of two rotations. In Proceedings of the 7th International Poplar Symposium, Buenos Aires, Argentina, 28 October4 November 2018.

158. González, I.; Sixto, H.; Rodríguez-Soalleiro, R.; Oliveira, N. Nutrient Contribution of Litterfall in a Short Rotation Plantation of Pure or Mixed Plots of Populus alba L. and Robinia pseudoacacia L. Forests 2020, 11, 1133. [CrossRef]

159. Grünewald, H.; Böhm, C.; Quinkenstein, A.; Grundmann, P.; Eberts, J.; von Wühlisch, G. Robinia pseudoacacia L.: A lesser known tree species for biomass production. Bioenergy Res. 2009, 2, 123-133. [CrossRef]

160. DeBell, D.S.; Harrington, C.A. Deploying genotypes in short-rotation plantations: Mixtures and pure cultures of clones and species. For. Chron. 1993, 69, 705-713. [CrossRef]

161. DeBell, D.S.; Harrington, C.A. Productivity of Populus in monoclonal and polyclonal blocks at three spacings. Can. J. For. Res. 1997, 27, 978-985. [CrossRef]

162. Miot, S.; Frey, P.; Pinon, J. Varietal mixture of poplar clones: Effects on infection by Melampsora larici-populina and on plant growth. Eur. J. For. Pathol. 1999, 29, 411-423. [CrossRef]

163. Elferjani, R.; DesRochers, A.; Tremblay, F. Effects of mixing clones on hybrid poplar productivity, photosynthesis and root development in northeastern Canadian plantations. For. Ecol. Manag. 2014, 327, 157-166. [CrossRef]

164. Dillen, M.; Vanhellemont, M.; Verdonckt, P.; Maes, W.H.; Steppe, K.; Verheyen, K. Productivity, stand dynamics and the selection effect in a mixed willow clone short rotation coppice plantation. Biomass Bioenergy 2016, 87, 46-54. [CrossRef]

165. Schweier, J.; Arranz, C.; Nock, C.A.; Jaeger, D.; Scherer-Lorenzen, M. Impact of increased genotype or species diversity in Short Rotation Coppice on biomass production and wood characteristics. Bioenergy Res. 2019, 12, 497-508. [CrossRef]

166. Broeckx, L.S.; Verlinden, M.S.; Ceulemans, R. Establishment and two-year growth of a bio-energy plantation with fast-growing Populus trees in Flanders (Belgium): Effects of genotype and former land use. Biomass Bioenergy 2012, 42, 151-163. [CrossRef] 
167. Morhart, C.; Sheppard, J.; Seidl, F.; Spiecker, H. Influence of different tillage systems and weed treatments in the establishment year on the final biomass production of short rotation coppice poplar. Forests 2013, 4, 849. [CrossRef]

168. Albertsson, J.; Hansson, D.; Bertholdsson, N.O.; Åhman, I. Site-related set-back by weeds on the establishment of 12 biomass willow clones. Weed Res. 2014, 54, 398-407. [CrossRef]

169. Heilman, P.E.; Xie, F.-G. Effects of nitrogen fertilization on leaf area, light interception, and productivity of short-rotation Populus trichocarpa $\times$ Populus deltoides hybrids. Can. J. For. Res. 1994, 24, 166-173. [CrossRef]

170. Ceulemans, R.; Deraedt, W. Production physiology and growth potential of poplars under short-rotation forestry culture. For. Ecol. Manag. 1999, 121, 9-23. [CrossRef]

171. Liberloo, M.; Calfapietra, C.; Lukac, M.; Godbold, D.; Luo, Z.-b.; Polle, A.; Hoosbeek, M.R.; Kull, O.; Marek, M.; Raines, C. Woody biomass production during the second rotation of a bio-energy Populus plantation increases in a future high $\mathrm{CO}_{2}$ world. Glob. Chang. Biol. 2006, 12, 1094-1106. [CrossRef]

172. Van Veen, J.; Breteler, H.; Olie, J.; Frissel, M. Nitrogen and energy balance of a short-rotation poplar forest system. Neth. J. Agri. Sci. 1981, 29, 163-172. [CrossRef]

173. Coleman, M.; Tolsted, D.; Nichols, T.; Johnson, W.D.; Wene, E.G.; Houghtaling, T. Post-establishment fertilization of Minnesota hybrid poplar plantations. Biomass Bioenergy 2006, 30, 740-749. [CrossRef]

174. Stanturf, J.A.; Van Oosten, C.; Netzer, D.A.; Coleman, M.D.; Portwood, C.J. Ecology and silviculture of poplar plantations. In Poplar Culture in North America; Dickmann, D.I., Isebrands, J.G., Eckenwalder, J.E., Richardson, J., Eds.; NRC Research Press: Ottawa, ON, Canada, 2002; pp. 153-206.

175. Yan, X.-L.; Dai, T.-F.; Zhao, D.; Jia, L.-M. Combined surface drip irrigation and fertigation significantly increase biomass and carbon storage in a Populus $\times$ euramericana cv. Guariento plantation. J. For. Res. 2016, 21, 280-290. [CrossRef]

176. Euring, D.; Löfke, C.; Teichmann, T.; Polle, A. Nitrogen fertilization has differential effects on $\mathrm{N}$ allocation and lignin in two Populus species with contrasting ecology. Trees 2012, 26, 1933-1942. [CrossRef]

177. Coleman, M.D.; Dickson, R.E.; Isebrands, J. Growth and physiology of aspen supplied with different fertilizer addition rates. Physiol. Plant. 1998, 103, 513-526. [CrossRef]

178. Paris, P.; Mareschi, L.; Sabatti, M.; Tosi, L.; Scarascia-Mugnozza, G. Nitrogen removal and its determinants in hybrid Populus clones for bioenergy plantations after two biennial rotations in two temperate sites in northern Italy. iForest 2015, 8, 657-665. [CrossRef]

179. Rennenberg, H.; Wildhagen, H.; Ehlting, B. Nitrogen nutrition of poplar trees. Plant Biol. 2010, 12, $275-291$. [CrossRef] [PubMed]

180. Wang, Y.; Xi, B.; Bloomberg, M.; Moltchanova, E.; Li, G.; Jia, L. Response of diameter growth, biomass allocation and $\mathrm{N}$ uptake to $\mathrm{N}$ fertigation in a triploid Populus tomentosa plantation in the North China Plain: Ontogenetic shift does not exclude plasticity. Eur. J. Forest Res. 2015, 134, 889-898. [CrossRef]

181. Gruber, N.; Galloway, J.N. An Earth-system perspective of the global nitrogen cycle. Nature 2008, $451,293$. [CrossRef] [PubMed]

182. Erisman, J.W.; van Grinsven, H.; Leip, A.; Mosier, A.; Bleeker, A. Nitrogen and biofuels; an overview of the current state of knowledge. Nut. Cycl. Agroecosyst. 2010, 86, 211-223. [CrossRef]

183. Heller, M.C.; Keoleian, G.A.; Volk, T.A. Life cycle assessment of a willow bioenergy cropping system. Biomass Bioenergy 2003, 25, 147-165. [CrossRef]

184. Sugiura, A.; Tyrrel, S.; Seymour, I.; Burgess, P. Water Renew systems: Wastewater polishing using renewable energy crops. Water Sci. Technol. 2008, 57, 1421-1428. [CrossRef]

185. Werner, A.; McCracken, A. The use of short rotation coppice poplar and willow for the bioremediation of sewage effluent. Asp. Appl. Biol. 2008, 317-324.

186. Dimitriou, I.; Rosenqvist, H. Sewage sludge and wastewater fertilisation of Short Rotation Coppice (SRC) for increased bioenergy production-Biological and economic potential. Biomass Bioenergy 2011, 35, 835-842. [CrossRef]

187. Martínez-Hernández, V.; Leal, M.; Meffe, R.; De Miguel, Á.; Alonso-Alonso, C.; De Bustamante, I.; Lillo, J.; Martín, I.; Salas, J. Removal of emerging organic contaminants in a poplar vegetation filter. J. Hazard. Mater. 2018, 342, 482-491. [CrossRef] [PubMed] 
188. Pradana, R.; Sixto, H.; González, B.; Demaria, I.; González, I.; Martínez-Hernández, V.; de Bustamante, I. Poplar Vegetation Filters for the beer industry: Wastewater treatment combined with biomass production. In Proceedings of the XI Congreso Ibérico de Gestión y Planificación del Agua, Madrid, Spain, 3-5 September 2020.

189. Paniagua, S.; Escudero, L.; Escapa, C.; Coimbra, R.N.; Otero, M.; Calvo, L.F. Effect of waste organic amendments on Populus sp biomass production and thermal characteristics. Renew. Energy 2016, 94, 166-174. [CrossRef]

190. Sixto, H.; Garasa, M.H.; Ciria, P.C.; Garcia, J.C.; de Vinas, I.C.R. Manual de Cultivo de Populus spp. Para la Producción de Biomasa Con Fines Energéticos. 2010. Available online: https://agris.fao.org/agris-search/ search.do?recordID=XF2015034695 (accessed on 15 November 2020).

191. Kollert, W. La populicultura en Europa y España. In Proceedings of the II Simposio del chopo, Valladolid, Spain, 17-19 October 2018.

192. Newcombe, G.; Ostry, M.; Hubbes, M.; Périnet, P.; Mottet, M.-J. Poplar diseases. In Poplar Culture in North America; Dickmann, D.I., Isebrands, J.G., Eckenwalder, J.E., Richardson, J., Eds.; NRC Research Press: Ottawa, ON, Canada, 2001; pp. 249-276. [CrossRef]

193. Coyle, D.R.; Nebeker, T.E.; Hart, E.R.; Mattson, W.J. Biology and management of insect pests in North American intensively managed hardwood forest systems. Annu. Rev. Entomol. 2005, 50, 1-29. [CrossRef] [PubMed]

194. Duplessis, S.; Major, I.; Martin, F.; Séguin, A. Poplar and pathogen interactions: Insights from Populus genome-wide analyses of resistance and defense gene families and gene expression profiling. Crit. Rev. Plant Sci. 2009, 28, 309-334. [CrossRef]

195. Broderick, N.A.; Vasquez, E.; Handelsman, J.; Raffa, K.F. Effect of clonal variation among hybrid poplars on susceptibility of gypsy moth (Lepidoptera: Lymantriidae) to Bacillus thuringiensis subsp. kurstaki. J. Econ. Entomol. 2010, 103, 718-725. [CrossRef]

196. Steenackers, J.; Steenackers, M.; Steenackers, V.; Stevens, M. Poplar diseases, consequences on growth and wood quality. Biomass Bioenergy 1996, 10, 267-274. [CrossRef]

197. Soria, S. Patología de las Choperas; Caja Rural del Duero. Colegio de Ingenieros de Montes: Valladolid, Spain, 1992; pp. 61-75.

198. Nordman, E.E.; Robison, D.J.; Abrahamson, L.P.; Volk, T.A. Relative resistance of willow and poplar biomass production clones across a continuum of herbivorous insect specialization: Univariate and multivariate approaches. For. Ecol. Manag. 2005, 217, 307-318. [CrossRef]

199. Isebrands, J.G.; Richardson, J. Poplars and Willows: Trees for Society and the Environment; The Food and Agricultural Organization of the United Nations (FAO) and CAB International: Rome, Italy, 2014.

200. Bloemen, J.; Fichot, R.; Horemans, J.A.; Broeckx, L.S.; Verlinden, M.S.; Zenone, T.; Ceulemans, R. Water use of a multi-genotype poplar short-rotation coppice from tree to stand scale. Glob. Chang. Biol. Bioenegy 2016, 9, 370-384. [CrossRef]

201. Padró, A.; Orensanz, J. El Chopo y su Cultivo; Ministerio de Agricultura, Pesca y Alimentación (MAPA): Madrid, Spain, 1987.

202. Sixto, H.; Ruiz, V.; Grau, J.; Montoto, J. Primeros resultados de un ensayo de riego en vivero de planta de chopo. 1. In Proceedings of the 1er Simposio del Chopo, Zaragoza, Spain, 9-11 May 2001; pp. 159-166.

203. Sixto, H.; Grau, J.; González-Antoñanzas, F. Populicultura: Populus spp. e híbridos. Capítulo IV. In Compendio de Selvicultura Aplicada en España; Serrada, R., Montero, G., Reque, J.A., Eds.; Institulo Nacional de Investigación y Tecnología Agraria y Alimentaria, Ministerio de Educación y Ciencia: Madrid, Spain, 2008.

204. IPCC. Climate Change 2007: Impacts, adaptation and vulnerability. In Contribution of Working Group II to the Fourth Assessment Report of the Intergovernmental Panel on Climate Change; Cambridge University Press: Cambridge, UK, 2007; p. 976.

205. González-González, B.D.; Oliveira, N.; González, I.; Cañellas, I.; Sixto, H. Poplar biomass production in short rotation under irrigation: A case study in the Mediterranean. Biomass Bioenergy 2017, 107, 198-206. [CrossRef]

206. Hennig, A.; Kleinschmit, J.R.; Schoneberg, S.; Löffler, S.; Janßen, A.; Polle, A. Water consumption and biomass production of protoplast fusion lines of poplar hybrids under drought stress. Front. Plant Sci. 2015, 6, 330. [CrossRef] 
207. Liang, Z.-S.; Yang, J.-W.; Shao, H.-B.; Han, R.-L. Investigation on water consumption characteristics and water use efficiency of poplar under soil water deficits on the Loess Plateau. Colloid Surface B 2006, 53, $23-28$. [CrossRef] [PubMed]

208. Viger, M.; Smith, H.; Cohen, D.; Dewoody, J.; Trewin, H.; Steenackers, M.; Bastien, C.; Taylor, G. Adaptive mechanisms and genomic plasticity for drought tolerance identifed in European black poplar (Populus nigra L.). Tree Physiol. 2016, 36, 909-928. [CrossRef] [PubMed]

209. Niemczyk, M.; Hu, Y.; Thomas, B.R. Selection of Poplar Genotypes for Adapting to Climate Change. Forests 2019, 10, 1041. [CrossRef]

210. Garasa, M.J.H. Respuesta Anatómico Fisiológicas Frente a Estrés Hídrico en Plantaciones de Especies de Crecimiento Rápido Para la Producción de Biomasa. Ph.D. Thesis, Universidad Politécnica de Madrid, Madrid, Spain, 2015.

211. Navarro, A.; Facciotto, G.; Campi, P.; Mastrorilli, M. Physiological adaptations of five poplar genotypes grown under SRC in the semi-arid Mediterranean environment. Trees 2014, 28, 983-994. [CrossRef]

212. Navarro, A.; Portillo-Estrada, M.; Arriga, N.; Vanbeveren, S.P.; Ceulemans, R. Genotypic variation in transpiration of coppiced poplar during the third rotation of a short-rotation bio-energy culture. Glob. Chang. Biol. Bioenergy 2018, 10, 592-607. [CrossRef] [PubMed]

213. Navarro, A.; Portillo-Estrada, M.; Ceulemans, R. Identifying the best plant water status indicator for bio-energy poplar genotypes. Glob. Chang. Biol. Bioenergy 2020, 12, 426-444. [CrossRef]

214. Fuertes, A.; Oliveira, N.; Cañellas, I.; Sixto, H.; Rodríguez-Soalleiro, R. An economic overview of Populus spp. in Short Rotation Coppice systems under Mediterranean conditions: An assessment tool for decision-making. Sustain. Energy Rev. 2020. under review.

215. de Bustamante, I. Filtros verdes. Un sistema para la depuración y reutilización de aguas residuales. Tecnoambiente 1998, 79, 73-75.

216. de Miguel, Á.; Martínez-Hernández, V.; Leal, M.; González-Naranjo, V.; de Bustamante, I. Del residuo al recurso: Reutilización de aguas para riego de biocombustibles-From waste to resource: Reuse of water for biofuel irrigation. Water 2012, 79, 68-70.

217. Makeschin, F. Short rotation forestry in Central and Northern Europe-introduction and conclusions. For. Ecol. Manag. 1999, 121, 1-7.

218. Ciria, M.; Mazón, M.; Carrasco, J. Poplar productivity evolution on short rotation during three consecutive cycles in extreme continental climate. In Proceedings of the 2nd World Conference on Biomass for Energy, Industry and Climate Protection, Rome, Italy, 10-14 May 2004.

219. Laureysens, I.; Deraedt, W.; Indeherberge, T.; Ceulemans, R. Population dynamics in a 6-year old coppice culture of poplar. I. Clonal differences in stool mortality, shoot dynamics and shoot diameter distribution in relation to biomass production. Biomass Bioenerg 2003, 24, 81-95. [CrossRef]

220. Benetka, V.; Novotná, K.; Štochlová, P. Biomass production of Populus nigra L. clones grown in short rotation coppice systems in three different environments over four rotations. iForest 2014, 7, 233-239. [CrossRef]

221. Trnka, M.; Trnka, M.; Fialová, J.; Koutecky, V.; Fajman, M.; Zalud, Z.; Hejduk, S. Biomass production and survival rates of selected poplar clones grown under a short-rotation system on arable land. Plant Soil Environ. 2008, 54, 78-88. [CrossRef]

222. Berthelot, A. Mélange de clones en taillis à courtes rotations de peuplier: Influence sur la productivité et l'homogénéité des produits récoltés. Can. J. For. Res. 2001, 31, 1116-1126. [CrossRef]

223. Hartmann, L.; Lamersdorf, N. Site conditions, initial growth and nutrient and litter cycling of newly installed short rotation coppice and agroforestry systems. In Bioenergy from Dendromass for the Sustainable Development of Rural Areas; Manning, D.B., Bemmann, A., Bredemeier, N., Lamersdorf, N., Ammer, C., Eds.; Wiley-VCH Verlag GmbH \& Co. KGaA: Weinheim, Germany, 2015.

224. Kern, J.; Germer, S.; Ammon, C.; Balasus, A.; Bischoff, W.-A.; Schwarz, A.; Forstreuter, M.; Kaupenjohann, M. Environmental effects over the first $2 \frac{1}{2}$ rotation periods of a fertilised poplar short rotation coppice. Bioenergy Res. 2018, 11, 152-165. [CrossRef]

225. Cabrera, A.; Tozzini, C.; Espinoza, S.; Santelices, R.; Bonari, E. Cálculo del balance energético de una plantación de Populus deltoides clon Lux con fines energéticos en un sitio con ambiente mediterráneo. Bosque 2014, 35, 133-139. [CrossRef] 
226. Paris, P.; Mareschi, L.; Sabatti, M.; Pisanelli, A.; Ecosse, A.; Nardin, F.; Scarascia-Mugnozza, G. Comparing hybrid Populus clones for SRF across northern Italy after two biennial rotations: Survival, growth and yield. Biomass Bioenergy 2011, 35, 1524-1532. [CrossRef]

227. Stolarski, M.J.; Krzyżaniak, M.; Szczukowski, S.; Tworkowski, J.; Bieniek, A. Short rotation woody crops grown on marginal soil for biomass energy. Pol. J. Environ. Stud. 2014, 23, 1727-1739.

228. Proe, M.F.; Griffiths, J.H.; Craig, J. Effects of spacing, species and coppicing on leaf area, light interception and photosynthesis in short rotation forestry. Biomass Bioenergy 2002, 23, 315-326. [CrossRef]

229. Baraza, C. Cultivos demostrativos de chopos (Populus spp.) a altas densidades con fines energéticos realizados en Aragón. Primeros datos y resultados. In Proceedings of the $6^{\circ}$ Congreso Forestal Español, Vitoria, Spain, 10-14 June 2013.

230. Ciria, M.; González, E.; Mazon, P.; Carrasco, J. Influence of the rotation age and plant density on the composition and quality of poplar biomass. Biomass for energy and the environment. In Proceedings of the 9th European Bioenergy Conference and 1st European Energy from Biomass Technology Exhibition, Copenhagen, Denmark, 24-27 June 1996; pp. 968-973.

231. Eimil-Fraga, C.; Proupín-Castiñeiras, X.; Rodríguez-Añón, J.A.; Rodríguez-Soalleiro, R. Effects of Shoot Size and Genotype on Energy Properties of Poplar Biomass in Short Rotation Crops. Energies 2019, 12, 2051. [CrossRef]

232. Godino, M. Análisis de los Cultivos de Populus $\times$ euroamericana, Clon I-214, a Turnos Muy Cortos con Fines Energéticos. Ph.D. Thesis, Universidad Politécnica de Madrid, Madrid, Spain, 2005.

233. Ibarra, D.; Eugenio, M.E.; Cañellas, I.; Sixto, H.; Martín-Sampedro, R. Potential of different poplar clones for sugar production. Wood Sci. Technol. 2017, 51, 669-684. [CrossRef]

234. Martín-Sampedro, R.; Eugenio, M.E.; Cañellas, I.; Sixto, H.; Ibarra, D. Characterization of different poplar clones for sugar production. In Proceedings of the 1st International Workshop on Biorefinery of Lignocellulosic Materials, Cordoba, Spain, 9-12 June 2015.

235. Djomo, S.N.; Ac, A.; Zenone, T.; De Groote, T.; Bergante, S.; Facciotto, G.; Sixto, H.; Ciria Ciria, P.; Weger, J.; Ceulemans, R. Energy performances of intensive and extensive short rotation cropping systems for woody biomass production in the EU. Renew. Sustain. Energy Rev. 2015, 41, 845-854. [CrossRef]

236. Oliveira, N.; Pérez-Cruzado, C.; Sixto, H.; Cañellas, I.; Rodrígez-Soalleiro, R. Modelización alométrica para la estimación de biomasa en un cultivo energético experimental de chopo. In Proceedings of the II Encuentro Juventud Investigadora. Universidad de Santiago de Compostela, Santiago de Compostela, Spain, 29-31 January 2014.

237. San Miguel, G.; Corona, B.; Ruiz, D.; Landholm, D.; Laina, R.; Tolosana, E.; Sixto, H.; Cañellas, I. Environmental, energy and economic analysis of a biomass supply chain based on a poplar short rotation coppice in Spain. J. Clean. Prod. 2015, 94, 93-101. [CrossRef]

238. Valbuena-Castro, J.; Oliveira, N.; Rodríguez-Soalleiro, R.; Sixto, H.; Cañellas, I. Biomass productivity assessment at a clonal forest of Populus spp. at Valtierra (Navarra, Spain). In Proceedings of the Xth Young Researchers Meeting on Conservation and Sustainable Use of Forest Systems, Valsaín, Spain, 25-26 January 2016.

239. Valbuena-Castro, J.; Oliveira, N.; Rodríguez-Soalleiro, R.; Cañellas, I.; Sixto, H. Assessment of biomass productivity and effect of planting density in a short rotation coppice poplar plantation in the north of Spain. In Proceedings of the 25th Session of the International Poplar Commission, Berlin, Germany, 12-16 September 2016.

240. Picard, N.; Saint-André, L.; Henry, M. Manual for Building Tree Volume and Biomass Allometric Equations: From Field Measurement to Prediction; Food and Agricultural Organization of the United Nations and Centre de Coopération Internationale en Recherche Agronomique pour le Développement, Montpellier: Rome, Italy, 2012; p. 215.

241. Peichl, M.; Arain, M.A. Allometry and partitioning of above- and belowground tree biomass in an age-sequence of white pine forests. For. Ecol. Manag. 2007, 253, 68-80. [CrossRef]

242. Shaiek, O.; Loustau, D.; Trichet, P.; Meredieu, C.; Bachtobji, B.; Garchi, S.; Aouni, M.H.E. Generalized biomass equations for the main aboveground biomass components of maritime pine across contrasting environments. Ann. For. Sci. 2011, 68, 443-452. [CrossRef] 
243. Verwijst, T.; Lundkvist, A.; Edelfeldt, S.; Albertsson, J. Development of sustainable willow short rotation forestry in Northern Europe. In Biomass Now-Sustainable Growth and Use; Matovic, D.M.D., Ed.; BoD-Books on Demand: Croatia, 2013; pp. 479-502. Available online: https://books.google.com.hk/books?hl=zh-CN\& lr=\&id=WdmgDwAAQBAJ\&oi=fnd\&pg=PA479\&dq=Development + of + sustainable + willow + short + rotation+forestry+in+Northern+Europe\&ots=BJwT6IWByQ\&sig=TZ6h3-16e8g3YMKL1hdp77ywZ2w\& redir_esc $=y \& h l=z h-C N \&$ sourceid $=$ cndr\# $\mathrm{v}=$ onepage $\& \mathrm{q}=$ Development $\% 20$ of $\% 20$ sustainable $\% 20 \mathrm{willow} \%$ 20short\%20rotation\%20forestry\%20in\%20Northern\%20Europe\&f=false (accessed on 15 November 2020). [CrossRef]

244. Mosseler, A.; Major, J.; Labrecque, M.; Larocque, G. Allometric relationships in coppice biomass production for two North American willows (Salix spp.) across three different sites. For. Ecol. Manag. 2014, 320, 190-196. [CrossRef]

245. Verlinden, M.S.; Broeckx, L.S.; Ceulemans, R. First vs. second rotation of a poplar short rotation coppice: Above-ground biomass productivity and shoot dynamics. Biomass Bioenergy 2015, 73, 174-185. [CrossRef]

246. Cannell, M.; Willett, S. Shoot growth phenology, dry matter distribution and root: Shoot ratios of provenances of Populus trichocarpa, Picea sitchensis and Pinus contorta growing in Scotland. Silvae Genet. 1976, 25, 49-59.

247. Ruark, G.A.; Martin, G.L.; Bockheim, J.G. Comparison of constant and variable allometric ratios for estimating Populus tremuloides biomass. For. Sci. 1987, 33, 294-300. [CrossRef]

248. Oliveira, N.; Rodríguez-Soalleiro, R.; Hernández, M.J.; Cañellas, I.; Sixto, H.; Pérez-Cruzado, C. Improving biomass estimation in a Populus short rotation coppice plantation. For. Ecol. Manag. 2017, 391, 194-206. [CrossRef]

249. Di Matteo, G.; Sperandio, G.; Verani, S. Field performance of poplar for bioenergy in southern Europe after two coppicing rotations: Effects of clone and planting density. iForest 2012, 5, 224-229. [CrossRef]

250. Rock, J. Suitability of published biomass equations for aspen in Central Europe-Results from a case study. Biomass Bioenergy 2007, 31, 299-307. [CrossRef]

251. Coyle, D.R.; Coleman, M.D. Forest production responses to irrigation and fertilization are not explained by shifts in allocation. For. Ecol. Manag. 2005, 208, 137-152. [CrossRef]

252. Oliveira, N.; Rodríguez-Soalleiro, R.; Pérez-Cruzado, C.; Cañellas, I.; Sixto, H. On the genetic affinity of individual tree biomass allometry in poplar short rotation coppice. Bioenergy Res. 2017, 10, 525-535. [CrossRef]

253. Pérez-Cruzado, C.; Fehrmann, L.; Magdon, P.; Cañellas, I.; Sixto, H.; Kleinn, C. On the site-level suitability of biomass models. Environ. Modell. Softw. 2015, 73, 14-26. [CrossRef]

254. Zianis, D.; Muukkonen, P.; Mäkipää, R.; Mencuccini, M. Biomass and Stem Volume Equations for Tree Species in Europe; Tammer-Paino Oy: Tampere, Finland, 2005.

255. Dowell, R.C.; Gibbins, D.; Rhoads, J.L.; Pallardy, S.G. Biomass production physiology and soil carbon dynamics in short-rotation-grown Populus deltoides and P. deltoides $\times$ P. nigra hybrids. For. Ecol. Manag. 2009, 257, 134-142. [CrossRef]

256. Johansson, T.; Karacic, A. Increment and biomass in hybrid poplar and some practical implications. Biomass Bioenergy 2011, 35, 1925-1934. [CrossRef]

257. Zabek, L.M.; Prescott, C.E. Biomass equations and carbon content of aboveground leafless biomass of hybrid poplar in Coastal British Columbia. For. Ecol. Manag. 2006, 223, 291-302. [CrossRef]

258. Ben Brahim, M.; Gavaland, A.; Cabanettes, A. Generalized allometric regression to estimate biomass of Populus in short-rotation coppice. Scand. J. For. Res. 2000, 15, 171-176. [CrossRef]

259. Casella, E.; Sinoquet, H. A method for describing the canopy architecture of coppice poplar with allometric relationships. Tree Physiol. 2003, 23, 1153-1170. [CrossRef]

260. Berhongaray, G.; Janssens, I.A.; King, J.S.; Ceulemans, R. Fine root biomass and turnover of two fast-growing poplar genotypes in a short-rotation coppice culture. Plant Soil 2013, 373, 269-283. [CrossRef] [PubMed]

261. Berhongaray, G.; Verlinden, M.S.; Broeckx, L.S.; Ceulemans, R. Changes in belowground biomass after coppice in two Populus genotypes. For. Ecol. Manag. 2015, 337, 1-10. [CrossRef]

262. Oliveira, N.; Rodríguez-Soalleiro, R.; Pérez-Cruzado, C.; Cañellas, I.; Sixto, H.; Ceulemans, R. Above- and below-ground carbon accumulation and biomass allocation in poplar short rotation plantations under Mediterranean conditions. For. Ecol. Manag. 2018, 428, 57-65. [CrossRef]

263. Amichev, B.Y.; Johnston, M.; Van Rees, K.C.J. Hybrid poplar growth in bioenergy production systems: Biomass prediction with a simple process-based model (3PG). Biomass Bioenergy 2010, 34, 687-702. [CrossRef] 
264. Headlee, W.L.; Zalesny, R.S., Jr.; Donner, D.M.; Hall, R.B. Using a process-based model (3-PG) to predict and map hybrid poplar biomass productivity in Minnesota and Wisconsin, USA. BioEnergy Res. 2013, 6, 196-210. [CrossRef]

265. Hart, Q.J.; Prilepova, O.; Merz, J.R.; Bandaru, V.; Jenkins, B.M. Modeling poplar growth as a short rotation woody crop for biofuels. UC Davis: Institute of Transportation Studies: EScholarship. University of California. 2014. Available online: https://escholarship.org/uc/item/1cc1p27b (accessed on 15 November 2020).

266. Hart, Q.J.; Tittmann, P.W.; Bandaru, V.; Jenkins, B.M. Modeling poplar growth as a short rotation woody crop for biofuels in the Pacific Northwest. Biomass Bioenergy 2015, 79, 12-27. [CrossRef]

267. Beringer, T.; Lucht, W.; Schaphoff, S. Bioenergy production potential of global biomass plantations under environmental and agricultural constraints. Glob. Chang. Biol. Bioenergy 2011, 3, 299-312. [CrossRef]

268. Rodrigues, A.; Gonçalves, A.B.; Casquilho, M.; Gomes, A.A. A GIS-based evaluation of the potential of woody short rotation coppice (SRC) in Portugal aiming at co-firing and decentralized co-generation. Biomass Bioenergy 2020, 137, 105554. [CrossRef]

269. Djomo, S.N.; De Groote, T.; Gobin, A.; Ceulemans, R.; Janssens, I.A. Combining a land surface model with life cycle assessment for identifying the optimal management of short rotation coppice in Belgium. Biomass Bioenergy 2019, 121, 78-88. [CrossRef]

270. Castaño-Díaz, M.; Álvarez-Álvarez, P.; Tobin, B.; Nieuwenhuis, M.; Afif-Khouri, E.; Cámara-Obregón, A. Evaluation of the use of low-density LiDAR data to estimate structural attributes and biomass yield in a short-rotation willow coppice: An example in a field trial. Ann. For. Sci. 2017, 74, 69. [CrossRef]

271. Sánchez-González, M.; Durbán, M.; Lee, D.-J.; Cañellas, I.; Sixto, H. Smooth additive mixed models for predicting aboveground biomass. J. Agric. Biol. Environ. Stat. 2017, 22, 23-41. [CrossRef]

272. Barrio-Anta, M.; Sixto-Blanco, H.; Viñas, I.C.-R.D.; Castedo-Dorado, F. Dynamic growth model for I-214 poplar plantations in the northern and central plateaux in Spain. Forest Ecol. Manag. 2008, 255, 1167-1178. [CrossRef]

273. de Ron, D.S.; Pérez-Cruzado, C.; González-Estevez, V.; Rodríguez-Soalleiro, R.; Cañellas, I.; Sixto, H. Visor cartográfico sobre producción de biomasa de chopo (Populus spp.) con fines energéticos en España. In Proceedings of the $6^{\circ}$ Congreso Forestal Español, Vitoria-Gasteiz, Spain, 10-14 June 2013.

274. Gasol, C.M.; Gabarrell, X.; Rigola, M.; González-García, S.; Rieradevall, J. Environmental assessment:(LCA) and spatial modelling (GIS) of energy crop implementation on local scale. Biomass Bioenergy 2011, 35, 2975-2985. [CrossRef]

275. Andújar, D.; Fernández-Quintanilla, C.; Dorado, J. Matching the best viewing angle in depth cameras for biomass estimation based on poplar seedling geometry. Sensors 2015, 15, 12999-13011. [CrossRef] [PubMed]

276. Andújar, D.; Rosell-Polo, J.R.; Sanz, R.; Rueda-Ayala, V.; Fernández-Quintanilla, C.; Ribeiro, A.; Dorado, J. A LiDAR-based system to assess poplar biomass. Gesunde Pflanz. 2016, 68, 155-162. [CrossRef]

277. Oliveira, N.; Sixto, H.; Cañellas, I.; Rodríguez-Soalleiro, R.; Pérez-Cruzado, C. Productivity model and reference diagram for short rotation biomass crops of poplar grown in Mediterranean environments. Biomass Bioenergy 2015, 72, 309-320. [CrossRef]

278. Isikgor, F.H.; Becer, C.R. Lignocellulosic biomass: A sustainable platform for the production of bio-based chemicals and polymers. Polym. Chem. 2015, 6, 4497-4559. [CrossRef]

279. Sannigrahi, P.; Ragauskas, A.J.; Tuskan, G.A. Poplar as a feedstock for biofuels: A review of compositional characteristics. Biofuel. Bioprod. Biorefin. 2010, 4, 209-226. [CrossRef]

280. Bioplat. Manual Sobre las Biorrefinerías en España; Bioplat, Suschem y Agencia estatal de investigación del Ministerio de Economía, Industría y Competitividad: Madrid, Spain, 2017.

281. Tharakan, P.J.; Volk, T.A.; Abrahamson, L.P.; White, E.H. Energy feedstock characteristics of willow and hybrid poplar clones at harvest age. Biomass Bioenerg 2003, 25, 571-580. [CrossRef]

282. Obernberger, I.; Brunner, T.; Bärnthaler, G. Chemical properties of solid biofuels-significance and impact. Biomass Bioenergy 2006, 30, 973-982. [CrossRef]

283. Elder, T.; Groom, L.H. Pilot-scale gasification of woody biomass. Biomass Bioenergy 2011, 35, 3522-3528. [CrossRef]

284. Kloss, S.; Zehetner, F.; Dellantonio, A.; Hamid, R.; Ottner, F.; Liedtke, V.; Schwanninger, M.; Gerzabek, M.H.; Soja, G. Characterization of slow pyrolysis biochars: Effects of feedstocks and pyrolysis temperature on biochar properties. J. Environ. Qual. 2012, 41, 990-1000. [CrossRef] [PubMed] 
285. Bridgwater, T. Challenges and opportunities in fast pyrolysis of biomass: Part I. Johnson Matthey Technol. Rev. 2018, 62, 118-130. [CrossRef]

286. Raud, M.; Kikas, T.; Sippula, O.; Shurpali, N.J. Potentials and challenges in lignocellulosic biofuel production technology. Renew. Sustain. Energy Rev. 2019, 111, 44-56. [CrossRef]

287. Moreno, A.D.; Alvira, P.; Ibarra, D.; Tomás-Pejó, E. Production of ethanol from lignocellulosic biomass. In Production of Platform Chemicals from Sustainable Resources; Springer: Berlin/Heidelberg, Germany, 2017; pp. $375-410$.

288. González-García, S.; Dias, A.C.; Clermidy, S.; Benoist, A.; Bellon Maurel, V.; Gasol, C.M.; Gabarrell, X.; Arroja, L. Comparative environmental and energy profiles of potential bioenergy production chains in Southern Europe. J. Clean. Prod. 2014, 76, 42-54. [CrossRef]

289. Martínez, M.; Duret, X.; Minh, D.P.; Nzihou, A.; Lavoie, J. Conversion of lignocellulosic biomass in biobutanol by a NOVEL thermal process. Int. J. Energy Prod. Manag. 2019, 4, 298-310. [CrossRef]

290. Zhang, Y.; Xia, C.; Lu, M.; Tu, M. Effect of overliming and activated carbon detoxification on inhibitors removal and butanol fermentation of poplar prehydrolysates. Biotechnol. Biofuels 2018, 11, 178. [CrossRef]

291. Krzyżaniak, M.; Stolarski, M.J.; Warmiński, K. Life cycle assessment of poplar production: Environmental impact of different soil enrichment methods. J. Clean. Prod. 2019, 206, 785-796. [CrossRef]

292. Díaz-Ramírez, M.; Nogués, F.S.; Royo, J.; Rezeau, A. Combustion Behavior of Novel Energy Crops in Domestic Boilers: Poplar and Brassica Experiences. In Alternative Energies; Springer: Berlin/Heidelberg, Germany, 2013; pp. 27-45.

293. Díaz-Ramírez, M.C. Grate-Fired Energy Crop Conversion: Experiences with Brassica Carinata and Populus sp.; Springer: Berlin/Heidelberg, Germany, 2015.

294. Díaz-Ramírez, M.; Boman, C.; Sebastián, F.; Royo, J.; Xiong, S.; Boström, D. Environmental performance of three novel opportunity biofuels: Poplar, brassica and cassava during fixed bed combustion. In Herbaceous Platns: Cultivation Methods, Grazing and Environmental Impacts; Wallner, F., Ed.; Nova Science Publishers: Hauppauge, NY, USA, 2013.

295. Santos, J.I.; Fillat, Ú.; Martín-Sampedro, R.; Ballesteros, I.; Manzanares, P.; Ballesteros, M.; Eugenio, M.E.; Ibarra, D. Lignin-enriched fermentation residues from bioethanol production of fast-growing poplar and forage sorghum. Bioresources 2015, 10, 5215-5232. [CrossRef]

296. Paniagua, S.; García-Pérez, A.I.; Calvo, L.F. Biofuel consisting of wheat straw-poplar wood blends: Thermogravimetric studies and combustion characteristic indexes estimation. Biomass Convers. Biorefin. 2019, 9, 433-443. [CrossRef]

297. Serrano, D.; Coronado, J.M.; Melero, J.A. Conversion of cellulose and hemicellulose into platform molecules: Chemical routes. In Biorefinery: From Biomass to Chemicals and Fuels; Aresta, M., Dibenedetto, A., Dumeignil, F., Eds.; The Gruyter: Berlin/Boston, Germany, 2012; pp. 123-140.

298. Berrueco Martínez, B.; Langa Morales, E.; Maestro Tejada, C.; Mainar Fernández, A.M.; Urieta Navarro, J.S. Potencial de las yemas de chopo como fuente de compuestos bioactivos: Actividad antioxidante de sus extractos supercríticos. In Proceedings of the $5^{\circ}$ Congreso Forestal Español, Ávila, Spain, 21-25 September 2009.

299. Martín-Sampedro, R.; Santos, J.I.; Fillat, Ú.; Wicklein, B.; Eugenio, M.E.; Ibarra, D. Characterization of lignins from Populus alba L. generated as by-products in different transformation processes: Kraft pulping, organosolv and acid hydrolysis. Int. J. Biol. Macromol. 2019, 126, 18-29. [CrossRef]

300. McKendry, P. Energy production from biomass (part 1): Overview of biomass. Bioresour. Technol. 2002, 83, 37-46. [CrossRef]

301. Kenney, W.A.; Sennerby-Forsse, L.; Layton, P. A review of biomass quality research relevant to the use of poplar and willow for energy conversion. Biomass 1990, 21, 163-188. [CrossRef]

302. Fernández, M.J.; Ciria, P.; Barro, R.; Losada, J.; Pérez, J.; Sixto, H.; Carrasco, J.E. Quality of the biomass produced in Short Rotation Coppices of poplar, willow, black locust and sycamore in two different spanish locations. Biomass Resour. 2013, 134-139. [CrossRef]

303. Monedero, E.; Hernández, J.J.; Cañellas, I.; Otero, J.M.; Sixto, H. Thermochemical and physical evaluation of poplar genotypes as short rotation forestry crops for energy use. Energy Convers. Manag. 2016, 129, 131-139. [CrossRef]

304. Pliura, A.; Zhang, S.; MacKay, J.; Bousquet, J. Genotypic variation in wood density and growth traits of poplar hybrids at four clonal trials. For. Ecol. Manag. 2007, 238, 92-106. [CrossRef] 
305. Marcos, F.; García, R.; García, F.; Godino, M.; Relova, I.; Villegas, S. Caracterización energética de la biomasa de chopo (Populus x euramericana I-214). In Proceedings of the $4^{\circ}$ Congreso Forestal Español, Zaragoza, Spain, 26-30 September 2005.

306. Marcos, F.; Godino, M.; García, F.; Izquierdo, I.; Villegas, S. Tallares de chopo I-214 a turnos muy cortos. In Proceedings of the $4^{\circ}$ Congreso Forestal Español, Zaragoza, Spain, 26-30 September 2005.

307. Marcos, F.; Villegas, S.; García, F.; Godino, M. Caracterización energética de la biomasa de chopo (Populus x euramericana I-214) en turnos muy cortos. Rev. Energ. 2006. Available online: file:///C:/Users/MDPI/ AppData/Local/Temp/16541-Texto\%20del\%20art\%C3\%ADculo-16533-1-10-20140611-1.pdf (accessed on 15 November 2020).

308. Llorente, M.J.F.; Laplaza, J.M.M.; Cuadrado, R.E.; García, J.E.C. Ash behaviour of lignocellulosic biomass in bubbling fluidised bed combustion. Fuel 2006, 85, 1157-1165. [CrossRef]

309. Bartolomé, C.; Gil, A. Ash deposition and fouling tendency of two energy crops (cynara and poplar) and a forest residue (pine chips) co-fired with coal in a pulverized fuel pilot plant. Energy Fuel 2013, 27, 5878-5889. [CrossRef]

310. Díaz-Ramírez, M.; Boman, C.; Sebastián, F.; Royo, J.; Xiong, S.; Boström, D. Ash characterization and transformation behavior of the fixed-bed combustion of novel crops: Poplar, brassica, and cassava fuels. Energy Fuel 2012, 26, 3218-3229. [CrossRef]

311. Díaz-Ramírez, M.; Sebastián, F.; Royo, J.; Rezeau, A. Influencing factors on $\mathrm{NO}_{X}$ emission level during grate conversion of three pelletized energy crops. Appl. Energy 2014, 115, 360-373. [CrossRef]

312. Cai, J.; He, Y.; Yu, X.; Banks, S.W.; Yang, Y.; Zhang, X.; Yu, Y.; Liu, R.; Bridgwater, A.V. Review of physicochemical properties and analytical characterization of lignocellulosic biomass. Renew. Sustain. Energy Rev. 2017, 76, 309-322. [CrossRef]

313. Vassilev, S.V.; Baxter, D.; Andersen, L.K.; Vassileva, C.G. An overview of the chemical composition of biomass. Fuel 2010, 89, 913-933. [CrossRef]

314. Bartolomé, C.; Gil, A. Emissions during co-firing of two energy crops in a PF pilot plant: Cynara and poplar. Fuel Process. Technol. 2013, 113, 75-83. [CrossRef]

315. González-García, S.; Moreira, M.T.; Feijoo, G.; Murphy, R.J. Comparative life cycle assessment of ethanol production from fast-growing wood crops (black locust, eucalyptus and poplar). Biomass Bioenergy 2012, 39, 378-388. [CrossRef]

316. Dallemand, J.F.; Petersen, J.E.; Karp, A. Short Rotation Forestry, Short Rotation Coppice and Perennial Grasses in the European Union: Agro-Environmental Aspects, Present Use and Perspectives; JRC Scientific and Technical Reports: Luxembourg, 2007; pp. 106-111.

317. Dimitriou, I.; Rutz, D. Sustainability Criteria and Recommendations for Short Rotation Woody Crops; IEE Project SRCplus: Munich, Germany, 2014.

318. Zalesny, R.S., Jr.; Donner, D.M.; Coyle, D.R.; Headlee, W.L. An approach for siting poplar energy production systems to increase productivity and associated ecosystem services. For. Ecol. Manag. 2012, 284, 45-58. [CrossRef]

319. Schweier, J.; Molina-Herrera, S.; Ghirardo, A.; Grote, R.; Díaz-Pinés, E.; Kreuzwieser, J.; Haas, E.; Butterbach-Bahl, K.; Rennenberg, H.; Schnitzler, J.P. Environmental impacts of bioenergy wood production from poplar short-rotation coppice grown at a marginal agricultural site in Germany. Bioenergy 2017, 9, 1207-1221. [CrossRef]

320. El Kasmioui, O.; Ceulemans, R. Financial analysis of the cultivation of poplar and willow for bioenergy. Biomass Bioenergy 2012, 43, 52-64. [CrossRef]

321. Manzone, M.; Bergante, S.; Facciotto, G. Energy and economic evaluation of a poplar plantation for woodchips production in Italy. Biomass Bioenergy 2014, 60, 164-170. [CrossRef]

322. Gasol, C. Environmental and Economic Integrated Assessment of Local Energy Crops Production in Southern Europe. Ph.D. Thesis, Universitat Autònoma de Barcelona, Barcelona, Spain, 2009.

323. González-García, S.; Gasol, C.M.; Gabarrell, X.; Rieradevall, J.; Moreira, M.T.; Feijoo, G. Environmental profile of ethanol from poplar biomass as transport fuel in Southern Europe. Renew. Energy 2010, 35, 1014-1023. [CrossRef]

324. Blanc, S.; Gasol, C.M.; Martínez-Blanco, J.; Muñoz, P.; Coello, J.; Casals, P.; Mosso, A.; Brun, F. Economic profitability of agroforestry in nitrate vulnerable zones in Catalonia (NE Spain). Span. J. Agric. Res. 2019, 17, 16. [CrossRef] 
325. Butnar, I.; Rodrigo, J.; Gasol, C.M.; Castells, F. Life-cycle assessment of electricity from biomass: Case studies of two biocrops in Spain. Biomass Bioenergy 2010, 34, 1780-1788. [CrossRef]

326. Gasol, C.M.; Gabarrell, X.; Anton, A.; Rigola, M.; Carrasco, J.; Ciria, P.; Rieradevall, J. LCA of poplar bioenergy system compared with Brassica carinata energy crop and natural gas in regional scenario. Biomass Bioenergy 2009, 33, 119-129. [CrossRef]

327. Relaño, R.L.; Esteban, E.T.; Rodríguez, S.J.H. Economic and Life Cycle Assessment of integrated wood and chips harvesting from hybrid poplar plantations in the Genil Valley (Spain). Comparison with chips harvesting from Poplar SRCs. In Proceedings of the From Theory to Practice: Challenges for Forest Engineering, Warsaw, Poland, 4-7 September 2016; p. 249.

328. Gasol, C.M.; Martínez, S.; Rigola, M.; Rieradevall, J.; Anton, A.; Carrasco, J.; Ciria, P.; Gabarrell, X. Feasibility assessment of poplar bioenergy systems in the Southern Europe. Renew. Sustain. Energy Rev. 2009, 13, 801-812. [CrossRef]

329. Li, W.; Ciais, P.; Makowski, D.; Peng, S. A global yield dataset for major lignocellulosic bioenergy crops based on field measurements. Sci. Data 2018, 5, 180169. [CrossRef] [PubMed]

330. Don, A.; Osborne, B.; Hastings, A.; Skiba, U.; Carter, M.S.; Drewer, J.; Flessa, H.; Freibauer, A.; Hyvönen, N.; Jones, M.B. Land-use change to bioenergy production in Europe: Implications for the greenhouse gas balance and soil carbon. Glob. Chang. Biol. Bioenergy 2012, 4, 372-391. [CrossRef]

331. Dimitriou, I.; Rutz, D. Sustainable Short Rotation Coppice: A Handbook; Rutz, D., Ed.; WIP Renewable Energies: Munich, Germany, 2015.

332. Rowe, R.L.; Street, N.R.; Taylor, G. Identifying potential environmental impacts of large-scale deployment of dedicated bioenergy crops in the UK. Renew. Sustain. Energy Rev. 2009, 13, 271-290. [CrossRef]

333. Griffiths, N.A.; Rau, B.M.; Vaché, K.B.; Starr, G.; Bitew, M.M.; Aubrey, D.P.; Martin, J.A.; Benton, E.; Jackson, C.R. Environmental effects of short-rotation woody crops for bioenergy: What is and isn't known. GCB-Bioenergy 2019, 11, 554-572. [CrossRef]

334. Pilipović, A.; Orlović, S.; Rončević, S.; Nikolić, N.; Župunski, M.; Spasojević, J. Results of selection of poplars and willows for water and sediment phytoremediation. Agric. For. 2015, 61, 205. [CrossRef]

335. Zalesny, R.S., Jr.; Headlee, W.L.; Gopalakrishnan, G.; Bauer, E.O.; Hall, R.B.; Hazel, D.W.; Isebrands, J.G.; Licht, L.A.; Negri, M.C.; Nichols, E.G. Ecosystem services of poplar at long-term phytoremediation sites in the Midwest and Southeast, United States. Willey Interdiscip. Rev. Energy Environ. 2019, 8, e349. [CrossRef]

336. Pandey, V.C.; Bajpai, O.; Singh, N. Energy crops in sustainable phytoremediation. Renew. Sustain. Energy Rev. 2016, 54, 58-73. [CrossRef]

337. Menéndez, J.; Loredo, J. Use of Reclaimed Surface Mines for Heat and Power Production. Int. J. Reneww. Energy Res. 2018, 3, 2367-9123.

338. Castaño-Díaz, M.; Barrio-Anta, M.; Afif-Khouri, E.; Cámara-Obregón, A. Willow short rotation coppice trial in a former mining area in Northern Spain: Effects of clone, fertilization and planting density on yield after five years. Forests 2018, 9, 154. [CrossRef]

339. de Bustamante, I.; Lillo, J.; Hernández, J.; Leal, M.; Meffe, R.; de Santiago, A.; Martínez-Hernández, V. El chopo como materia prima e instrumento medioambiental. In Proceedings of the II Simposio del chopo, Valladolid, Spain, 17-19 October 2018.

340. Rodríguez-Soalleiro, R.; Eimil-Fraga, C.; Gómez-García, E.; García-Villabrille, J.D.; Rojo-Alboreca, A.; Muñoz, F.; Oliveira, N.; Sixto, H.; Pérez-Cruzado, C. Exploring the factors affecting carbon and nutrient concentrations in tree biomass components in natural forests, forest plantations and short rotation forestry. For. Ecosyst. 2018, 5, 35. [CrossRef]

341. Oliveira, N.; Fuertes, A.; González, I.; de la Iglesia, J.P.; Cañellas, I.; Rodríguez-Soalleiro, R.; Sixto, H. Cultivo de chopo para la producción de biomasa: Estrategia de mitigación mediante la acumulación de carbono en las diferentes fracciones. In Proceedings of the VIII Remedia Workshop. Economía Circular como Catalizador de la Sostenibilidad Medioambiental del Sector Primario Español, Elche, Spain, 22-23 September 2020.

342. Rodríguez, F.; Serrano, L.; Aunós, A. El papel del chopo como sumidero de $\mathrm{CO}_{2}$ atmosférico. In Proceedings of the $4^{\circ}$ Congreso Forestal Español, Zaragoza, Spain, 26-30 September 2005.

343. Djomo, S.N.; Kasmioui, O.E.; Ceulemans, R. Energy and greenhouse gas balance of bioenergy production from poplar and willow: A review. Glob. Chang. Biol. Bioenergy 2011, 3, 181-197. [CrossRef] 
344. Pérez-Cruzado, C.; Mohren, G.M.; Merino, A.; Rodríguez-Soalleiro, R. Carbon balance for different management practices for fast growing tree species planted on former pastureland in southern Europe: A case study using the $\mathrm{CO}_{2}$ Fix model. Eur. J. For. Res. 2012, 131, 1695-1716. [CrossRef]

345. Pérez-Cruzado, C.; Mansilla-Salinero, P.; Rodríguez-Soalleiro, R.; Merino, A. Influence of tree species on carbon sequestration in afforested pastures in a humid temperate region. Plant Soil 2012, 353, 333-353. [CrossRef]

346. Verlinden, M.S.; Broeckx, L.S.; Zona, D.; Berhongaray, G.; De Groote, T.; Serrano, M.C.; Janssens, I.A.; Ceulemans, R. Net ecosystem production and carbon balance of an SRC poplar plantation during its first rotation. Biomass Bioenergy 2013, 56, 412-422. [CrossRef]

347. Ceulemans, R.; Horemans, J.A.; Vanbeveren, S.P.P. Short-rotation crops reduce greenhouse gas emissions and contribute to climate change mitigation. In Proceedings of the 7th International Poplar Symposium. New Bioeconomies: Exploring the potential role of Salicaceae, Buenos Aires, Argentina, 28 October4 November 2018.

348. Folch, A.; Ferrer, N. The impact of poplar tree plantations for biomass production on the aquifer water budget and base flow in a Mediterranean basin. Sci. Total Environ. 2015, 524, 213-224. [CrossRef]

349. MINETAD. La Energía en España 2016; Ministerio de Energía, Turismo y Agenda Digital: Madrid, Spain, 2017; p. 342.

350. Unión por la Biomasa. Balance Socioeconómico de las Biomasas en ESPAÑA 2017-2021; Unión por la Biomasa: Spain, 2018; p. 30. Available online: https://www.interempresas.net/Energia/Articulos/219726-Unionpor-Biomasa-presenta-〈T1\textquoterightBalance-socioeconomico-de-biomasas-en-Espana-2017-2021\T1\ textquoteright.html (accessed on 15 November 2020).

351. IDAE. Biomasa: Cultivos Energéticos; Instituto para la Diversificación y Ahorro de la Energía: Madrid, Spain, 2007.

352. Eurostat. Renewable Energy Statistics; European Commission: Brussels, Belgium, 2018.

353. WBA. Global Bioenergy Statistics; World Bioenergy Association: Stockholm, Sweden, 2019; p. 58.

354. APPA. Estudio del Impacto Macroeconómico de las ENERGÍAS Renovables en España; Asociación de Empresas de Energías Renovables: Spain, 2018; Available online: https://www.appa.es/wp-content/uploads/2018/10/ Estudio_del_impacto_Macroeconomico_de_las_energias_renovables_en_Espa\%C3\%B1a_2017.pdf (accessed on 15 November 2020).

Publisher's Note: MDPI stays neutral with regard to jurisdictional claims in published maps and institutional affiliations.

(C) 2020 by the authors. Licensee MDPI, Basel, Switzerland. This article is an open access article distributed under the terms and conditions of the Creative Commons Attribution (CC BY) license (http://creativecommons.org/licenses/by/4.0/). 